\title{
m-ISOMETRIC TRANSFORMATIONS OF HILBERT SPACE,II
}

Jim Agler and Mark Stankus

J. Agler

Department of Mathematics, Univ. Of Calif., San Diego

La Jolla, CA 92093-0112, U.S.A.

M. Stankus

Department of Mathematics, Univ. Of Calif., San Diego

La Jolla, CA 92093-0112, U.S.A.

In the first part of this series [AgSt], a model for operators satisfying the equation $\sum_{k=0}^{m}(-1)^{k}\left(\begin{array}{c}m \\ k\end{array}\right)\left(T^{*}\right)^{m-k} T^{m-k}=0$ was given as multiplication by $e^{i \phi}$ on a Hilbert space whose inner product is defined in terms of periodic distributions. In this paper and the next, wo relate this model theory for the case when $m=2$ to a disconjugacy theory for a subclass of Toeplitz operators of the type studied by Boutet de Monvel and Guillemin, classical function theoretic ideas on the Dirichlet space, and the theory of nonstationary stochastic processes.

\section{Table of Contents}

\section{Paper I.}

0. Introduction

1. The Elementary Operator Theory of $m$-Isometries

2. Distribution Differential Operators on the circle

3. A model for $m$-isometries

4. Smoothing applied to $m$-isometries

\section{Paper II.}

Introduction to Paper II

5. Brownian shifts, Brownian unitaries and Brownian isometries

6. A Disconjugacy Theorem for Matricial Toeplitz Operators

7. Another proof of the Lifting Theorem

\section{Paper III.}

Introduction to Paper III

8. A cyclic vector

9. Brownian Unitaries Revisited

10. Some remarks and Open Questions 


\section{Introduction}

In this paper we shall continue studying the bounded linear transformations $T$ of a complex Hilbert space $\mathcal{H}$ that satisfy an identity of the form

$$
T^{* m} T^{m}-\left(\begin{array}{c}
m \\
1
\end{array}\right) T^{*^{m-1}} T^{m-1}+\left(\begin{array}{c}
m \\
2
\end{array}\right) T^{*^{m-2}} T^{m-2}-\cdots+(-1)^{m}=0
$$

for a positive integer $m$ by specializing to the case of $m=2$. Operators $T$ satisfying the above equation are said to be $m$-isometries.

Some notation and results from the first part of this series [AgSt]: We now recall a few results and restate a few definitions from the first part of this series. When expedient, we shall specialize the results and definitions to the case of 2 -isometries.

If $T$ is a 2 -isometry, then $\Delta_{T}$ is defined to be the quantity $T^{*} T-1$. It was shown that if $T$ is a 2-isometry, then $\Delta_{T}$ is a positive operator and in the case that $T$ is finitely cyclic, $\Delta_{T}$ is compact.

A 2-isometry $T$ is said to be pure if it has no isometric direct summand.

DDO's of order $m$ are the objects which were used in Section 3 [AgSt] to give a distributional model for bicyclic invertible $(m+1)$-isometries. We now restate the definition of DDO.

Let $\mathcal{D}=C^{\infty}(\partial \mathbb{D})$, the Frechet space of infinitely differentiable functions on the unit circle. Let $\mathcal{D}^{\prime}$ denote the dual of $\mathcal{D}$, the space of distributions on the circle.

We define a linear operator $D: \mathcal{D} \rightarrow \mathcal{D}$ via the formula

$$
D \varphi=\frac{1}{i} \frac{d}{d \theta} \varphi
$$

Now recall that if $\beta \in \mathcal{D}^{\prime}$ and $\varphi \in \mathcal{D}$ then $\varphi \beta \in \mathcal{D}^{\prime}$ can be defined by

$$
\varphi \beta(\psi)=\beta(\varphi \psi), \quad \psi \in \mathcal{D}
$$

Thus, if $\beta \in \mathcal{D}^{\prime}, \beta$ can be regarded naturally as a map $\beta: \mathcal{D} \rightarrow \mathcal{D}^{\prime}$ by defining

$$
\beta(\varphi)=\varphi \beta
$$

Definition A distribution differential operator DDO of order 0 is a map $L: \mathcal{D} \rightarrow \mathcal{D}^{\prime}$ that has the form $L=\beta_{0}$ for some $\beta_{0} \in \mathcal{D}^{\prime}, \beta_{0} \neq 0$. A distribution differential operator DDO of order 1 is a map $L: \mathcal{D} \rightarrow \mathcal{D}^{\prime}$ that has the form $L=\beta_{1} D+\beta_{0}$ where $\beta_{0}, \beta_{1} \in \mathcal{D}^{\prime}$ and $\beta_{1} \neq 0$.

If $L$ is a DDO and $\varphi, \psi \in \mathcal{D}$, let us agree to define $\psi L \varphi: \mathcal{D} \rightarrow \mathcal{D}^{\prime}$ by

$$
(\psi L \varphi)(\chi)=\psi L(\varphi \chi), \quad \chi \in \mathcal{D}
$$


With this definition observe that if $L$ is a DDO of order $m$ and $\varphi, \psi \in \mathcal{D}$, then $\psi L \varphi$ is a DDO of order $\leq m$. The formal derivative of a DDO or order 1 is defined as follows.

Definition If $L=\beta_{1} D+\beta_{0}$ is a DDO of order 1 , then we define the DDO $\frac{d}{d D} L$ by setting

$$
\frac{d}{d D} L=\beta_{1}
$$

DTO's of order $m$ are the objects which were used in Section 3 to give a distributional model for cyclic $(m+1)$-isometries. We now restate the definition of DTO.

Define $\mathcal{D}_{a} \subset \mathcal{D}$ by

$$
\mathcal{D}_{a}=\{\varphi \in \mathcal{D}: \hat{\varphi}(n)=0 \text { if } n<0\}
$$

We let $P$ denote the canonical projection of $\mathcal{D}$ onto $\mathcal{D}_{a}$ defined by

$$
(P \varphi) \hat{(n)}=\left\{\begin{array}{cc}
\hat{\varphi}(n) & n \geq 0 \\
0 & n<0
\end{array} .\right.
$$

In like fashion, let $\mathcal{D}_{a}^{\prime}$ denote the space of analytic distributions defined by

$$
\mathcal{D}_{a}^{\prime}=\left\{u \in \mathcal{D}^{\prime}: u^{\prime}(n)=0 \quad \text { if } \quad n<0\right\}
$$

$\mathcal{D}_{a}^{\prime}$ can be regarded as the space of boundary values of analytic functions on $\mathbb{D}$ whose power series coefficients form a temperate sequence. We let $P$ denote the canonical projection of $\mathcal{D}^{\prime}$ onto $\mathcal{D}_{a}^{\prime}$ defined by

$$
(P u) \hat{(n)}=\left\{\begin{array}{cc}
\hat{u}(n) & n \geq 0 \\
0 & n<0
\end{array} .\right.
$$

Definition A distribution Toeplitz operator (DTO) is a linear mapping $A: \mathcal{D}_{a} \rightarrow \mathcal{D}_{a}^{\prime}$ that has the form

$$
A=P L \mid \mathcal{D}_{a}
$$

for some DDO $L$. If $A$ is a DTO, we define the order of $A$ to be the order of $L$ where $L$ is as above.

If $T \in \mathcal{L}(\mathcal{H})$ is a 2-isometry and $\gamma \in \mathcal{H}$, then since the unilateral array $\left\langle T^{k_{1}} \gamma, T^{k_{2}} \gamma\right\rangle$ is linear on diagonals it is natural to define the slope $\mu$ and intercept $\beta$ of $(T, \gamma)$ to be the elements of $\mathcal{D}^{\prime}$ defined by the formulas

$$
\hat{\mu(k)}=\left\{\begin{array}{cc}
\left\langle T^{k+1} \gamma, T \gamma\right\rangle-\left\langle T^{k} \gamma, \gamma\right\rangle & k \geq 0 \\
\left\langle T \gamma, T^{-k+1} \gamma\right\rangle-\left\langle\gamma, T^{-k} \gamma\right\rangle & k<0
\end{array}\right.
$$

and

$$
\hat{\beta}(k)=\left\{\begin{array}{cc}
\left\langle T^{k} \gamma, \gamma\right\rangle & k \geq 0 \\
\left\langle\gamma, T^{-k} \gamma\right\rangle & k<0
\end{array}\right.
$$


On the other hand there exist a pair of distributions $\beta_{0}$ and $\beta_{1}$ such that

$$
A=P\left(\beta_{1} D+\beta_{0}\right) \mid \mathcal{D}_{a}
$$

Furthermore, the formulas that relate the above distributions are given by $\beta_{1}=\mu$ and $\beta_{0}=\beta+(1-P)(D \mu)$.

The modelling of multicyclic (resp., multi bicylic invertible) $m$-isometries requires the use of matrices whose elements are DTO (resp.,DDO).

If $n_{1}$ and $n_{2}$ are positive integers, $\mathcal{D}^{n_{1}, n_{2}}$ denotes the space of $n_{1} \times n_{2}$ matrices with entries in $\mathcal{D}$. Likewise, let $\mathrm{DDO}_{m}^{n_{1}, n_{2}}$ denote the space of $n_{1} \times n_{2}$ matrices with DDO entries $L_{r s}$ with the order of $L_{r s} \leq m$ and let DTO $m_{m}^{n_{1}, n_{2}}$ denote the space of $n_{1} \times n_{2}$ matrices with DTO entries $A_{r s}$ with the order of $A_{r s} \leq m$

The proof of the lifting theorem which is proven is Section 7 requires the use of 2 classes of DDO (smooth and regular) which were introduced $n$ Section 4 . We now restate these definitions.

Definition If $L=\sum \beta_{\ell} D^{(\ell)} \in \mathrm{DDO}^{n, n}$, then $L$ is smooth if each $\beta_{\ell}$ is a matrix of smooth functions. $L$ is regular if $L$ is smooth and $\beta_{m}>0$ on $\partial \mathbb{D}$ (i.e., the $n \times n$ matrix $\beta_{m}\left(e^{i \theta}\right)$ is strictly positive definite for all $e^{i \theta} \in \partial \mathbb{D}$ where $\left.m=\operatorname{ord}(L)\right)$.

We remarked above that a distributional model for invertible $n$-bicyclic 2 -isometries was developed in Section 3 using DDO and DTO. The rest of this introduction will restate the notation which was used to obtain

(1) an element of $D D O^{n, n}$ from an invertible $n$-bicyclic $m$-isometry,

(2) an invertible $n$-bicyclic $m$-isometry from certain elements of $D D O^{n, n}$,

(3) an element of $D T O^{n, n}$ from an $n$-cyclic m-isometry and

(4) an $n$-cyclic $m$-isometry from certain elements of $D T O^{n, n}$.

If $\mathcal{H}$ is a Hilbert space, $T \in \mathcal{L}(\mathcal{H})$ is an invertible $m$-isometry and $\gamma \in \mathcal{H}$ is a nonzero vector, then $\gamma$ is bicyclic for $T \mid \bigvee\left\{T^{k} \gamma: k \in \mathbb{Z}\right\}$ and so there exists a uniquely associated Dirichlet operator given by Theorem 3.14 (of Section 3). We will denote this associated Dirichlet operator by $(T, \gamma)^{-}$.

If $T \in \mathcal{L}(\mathcal{H})$ is an $m$-isometry and $\gamma \in \mathcal{H}$ one can set

$$
\mathcal{H}_{\gamma}=\bigvee\left\{T^{k} \gamma: k \geq 0\right\}
$$

and

$$
T_{\gamma}=T \mid \mathcal{H}_{\gamma}
$$

With this setup, $T_{\gamma}$ is a cyclic $m$-isometry and $\gamma$ is a cyclic vector for $T_{\gamma}$. Accordingly by Theorem 3.23, there exists a unique analytic Dirichlet operator $A$ with the property that $\left(T_{\gamma}, \gamma\right)$ is unitarily equivalent to $\left(M_{A}, 1\right)$. We shall let $(T, \gamma)$ denote the unique DDO such that $A=P(T, \gamma)^{\wedge} \mid \mathcal{D}_{a}$. 
There is a certain amount of ambiguity in the $(T, \gamma)^{\wedge}$ notation. Observe that if $T \in$ $\mathcal{L}(\mathcal{H})$ is an invertible $m$-isometry and $\gamma \in \mathcal{H}$ then according to the remark following the proof of Theorem $3.14(T, \gamma)^{\wedge}$ is the unique Dirichlet operator such that

$$
\langle\varphi(T) \gamma, \psi(T) \gamma\rangle=(T, \gamma)^{\wedge}(\varphi)(\bar{\psi})
$$

for all $\varphi, \psi \in \mathcal{D}$. On the other hand, $(T, \gamma)^{\wedge}$ as just defined in previous paragraph has the defining property

$$
\begin{aligned}
\langle\varphi(T) \gamma, \psi(T) \gamma\rangle & =A(\varphi)(\bar{\psi}) \\
& =(T, \gamma) \hat{(}(\varphi)(\bar{\psi})
\end{aligned}
$$

for all $\varphi, \psi \in \mathcal{D}_{a}$. Recalling the remark following the proof of Lemma 3.3, we find that these two definitions of $(T, \gamma)^{\wedge}$ agree.

If $L \geq 0$ is a DDO, we define the form space of $L, H_{L}^{2}$, to be the completion of $\mathcal{D}$ with respect to the sesquilinear form $[\cdot, \cdot]_{L}$ on $\mathcal{D} \times \mathcal{D}$ given by

$$
[\varphi, \psi]=L(\varphi)(\bar{\psi})
$$

Specifically, one sets $\mathcal{M}_{L}=\left\{\varphi \in \mathcal{D}:[\varphi, \varphi]_{L}=0\right\}$, observes by the Cauchy-Schwarz inequality that $[\cdot, \cdot]_{L}$ induces a inner product on $\mathcal{D} / \mathcal{M}_{L}$, and lets $H_{L}^{2}$ denote the completion of $\mathcal{D} / \mathcal{M}_{L}$.

Likewise, if $A \geq 0$ is a DTO, we define the form space of $A, H_{A}^{2}$, to be the completion of $\mathcal{D}_{a}$ with respect to the sesquilinear form $[\cdot, \cdot]_{A}$ on $\mathcal{D}_{a} \times \mathcal{D}_{a}$ given by

$$
[\varphi, \psi]_{A}=A(\varphi)(\bar{\psi})
$$

If $L \geq 0$ is a DDO, then one can attempt to define a linear operator $M_{L}$ on $H_{L}^{2}$ via the formula

$$
\left(M_{L} \varphi\right)\left(e^{i \theta}\right)=e^{i \theta} \varphi\left(e^{i \theta}\right) .
$$

In general, this operator need not be well-defined on $\mathcal{D} / \mathcal{M}_{L}$ and, even if it is, may not extend to a bounded operator on $H_{L}^{2}$. The following definition precisely identifies those DDO $L$ with the property that $L \geq 0$ and $M_{L}$ is a well-defined bounded operator.

Definition A Dirichlet operator is a DDO $L$ with the property that either there exists a constant $c>1$ such that

$$
0 \leq L-c^{-2} e^{-i \theta} L e^{i \theta}
$$

or ord $(L)=0$ and $L \geq 0$.

Corresponding definitions of positivity for DTO $A$, a form space of $A$ and $M_{A}$ on $H_{A}^{2}$ are given in Section 3.

The following definition precisely identifies those DTO $A$ with the property that $A \geq 0$ and $M_{A}$ is a well-defined bounded operator. 
Definition An analytic Dirichlet operator is a DTO $A$ with the property that either there exists a constant $c>1$ such that

$$
0 \leq A-e^{-2} e^{-i \theta} A e^{i \theta}
$$

or $\operatorname{ord}(A)=0$ and $A \geq 0$.

$$
\begin{gathered}
\text { Finally, If } \psi=\left(\begin{array}{c}
\psi_{1} \\
\vdots \\
\psi_{n}
\end{array}\right) \in \mathcal{D}^{n, 1} \text {, we define } \psi^{*} \in \mathcal{D}^{1, n} \text { by setting } \\
\psi^{*}=\left(\bar{\psi}_{1}, \ldots, \bar{\psi}_{n}\right) .
\end{gathered}
$$

Brief Description of Sections 5 through 7: In Section 5 a certain class of 2-isometries motivated by statistical considerations is introduced, the Brownian unitaries. The irreducible Brownian unitaries correspond to the time shift operator on a scaled Brownian motion process and are referred to as Brownian shifts (Definition 5.5). Each Brownian shift is determined by a nonrandom rotation angle $\theta$ and a positive covariance scalar $\sigma$. It turns out that there is a direct integral theory for Brownian unitaries (Theorem 5.20), each is a direct integral with multiplicity over angles of Brownian shifts of a fixed covariance $\sigma$. The scalar $\sigma$ is referred to as the covariance of the Brownian unitary. Furthermore, the Brownian shifts in addition to their appearance as the time shift for perhaps the most ubiquitous of nonstationary Gaussian stochastic processes have elegent characterizations on the level of operator theory (Proposition 5.6) as well as within the field of DDO (Proposition 5.2). The principal result of Section 5, the Lifting Theorem for 2-Isometries (Theorem 5.80 ), asserts that the general 2-isometry $T$ with $\left\|\Delta_{T}\right\| \leq \sigma^{2}$ can be represented as the restriction to an invariant subspace of a Brownian unitary of covariance $\sigma$.

The proof of the Lifting Theorem in Section 5 consists of a synthesis of a proof of the Sz.Nagy Dilation Theorem and a proof of the familiar fact that isometries possess extensions to unitaries, and in particular, is purely algebraic in character. The original proof of the Lifting Theorem was analytic in character and derives from first principles the class of objects the 2-isometries are to be lifted to (i.e., the Brownian unitaries). The main analytic content here is a discongugacy theory for regular first order DDO with $L^{\infty}$ coefficients discussed in Section 6 (Lemmas 6.7 and 6.16 and Proposition 6.17).

A second class of operators discussed at length in Section 5 is the collection of direct integrals with multiplicity in $\theta$ of Brownian shifts with varying covariance which we refer to as Brownian isometries. This class not only arises naturally in Theorem 6.20 which studies the extensions of 2-isometries defined as multiplication by $e^{i \theta}$ on the form domain of Toeplitz operators (i.e., (0.5) with $m=1, \beta_{1}=\frac{d \theta}{2 \pi}$, and $\beta_{0}=w \frac{d \theta}{2 \pi}$ with $w \in L^{\infty}$ ) but also arises naturally in the general theory of Brownian unitary extensions which is studied in Section 9. Both the classes of Brownian unitaries and Brownian isometries possess elegant $C^{*}$-algebraic characterizations (Theorems 5.20 and 5.48). 


\section{§5. Brownian shifts, Brownian unitaries and Brownian isometries}

In this section, we introduce three special classes of 2-isometries - Brownian shifts, Brownian unitaries and Brownian isometries. The introduction of the first and second classes is motivated by statistical considerations as described below.

Let $S_{\sigma}=\left\{X_{0}, X_{1}, \ldots\right\}$ be a discretely observed mean 0 Brownian motion in $\mathbb{R}$ scaled so as to have covariance,

$$
E\left(X_{k_{1}} X_{k_{2}}\right)=1+\sigma^{2} \min \left\{k_{1}, k_{2}\right\}
$$

If we modify the process by rotating the real axis non-randomly in the complex plane by $\theta$ radians per unit time, then a new process $S_{\sigma, \theta}$ is obtained with covariance given by

$$
E\left(X_{k_{1}} \overline{X_{k_{2}}}\right)=e^{i\left(k_{1}-k_{2}\right) \theta}\left(1+\sigma^{2} \min \left\{k_{1}, k_{2}\right\}\right) .
$$

Stationary processes are characterized by the fact that the time shift operator $T$ defined on the Hilbert space associated with the process by requiring

$$
T X_{k}=X_{k+1}, \quad k \geq 0
$$

to be an isometry. What is the time shift operator on our modified Brownian motion process?

Proposition 5.2. If $T_{\sigma, \theta}$ denotes the time shift operator of the process $S_{\sigma, \theta}$, then $T_{\sigma, \theta}$ is unitarily equivalent to the block operator

$$
B_{\sigma, e^{i \theta}}=\left[\begin{array}{cc}
S & \sigma(1 \otimes 1) \\
0 & e^{i \theta}
\end{array}\right]
$$

acting on $H^{2} \oplus \mathbb{C}$ where $S$ is multiplication by $z$ on $H^{2}$ and $1 \otimes 1: \mathbb{C} \rightarrow H^{2}$ is defined by $(1 \otimes 1)(c)(z)=c, c \in \mathbb{C}, z \in \mathbb{D}$.

Proof. Let $\mathcal{H}$ denote the Hilbert space of the process $S_{\sigma, \theta}$. Thus, $\mathcal{H}$ is the completion of the formal linear combinations of the variables $X_{0}, X_{1}, \ldots$ with respect to the inner product $(\cdot, \cdot)$ given by

$$
\left(X_{k_{1}}, X_{k_{2}}\right)=E\left(X_{k_{1}} \overline{X_{k_{2}}}\right) .
$$

We densely define a linear map $U_{0}: \mathcal{H} \rightarrow H^{2} \oplus \mathbb{C}$ by requiring that

$$
U_{0}\left(X_{k}\right)=\left[\begin{array}{c}
\sigma \frac{z^{k}-e^{i k \theta}}{z-e^{i \theta}} \\
e^{i k \theta}
\end{array}\right],
$$


for each $k \geq 0$. First observe using (5.1), (5.3) and (5.4) that

$$
\left\langle U_{0}\left(X_{k_{1}}\right), U_{0}\left(X_{k_{2}}\right)\right\rangle=\left(X_{k_{1}}, X_{k_{2}}\right)
$$

a formula which implies that $U_{0}$ is isometric. Hence $U_{0}$ extends by continuity to an isometry $U$ defined on $\mathcal{H}$. Also, observe that

$$
\bigvee\left\{U_{0}\left(X_{k}\right): k \leq n\right\}=\left(\bigvee\left\{z^{k}: k<n\right\}\right) \Leftrightarrow \mathbb{C}
$$

for each $n \geq 0$. Thus, $U$ is a Hilbert space isomorphism.

Finally, note using (5.4) and the block form of $B_{\sigma, e^{2 \theta}}$, that

$$
U\left(X_{k}\right)=\left(B_{\sigma, e^{i \theta}}\right)^{k} U\left(X_{0}\right) \quad, \quad k \geq 0
$$

Thus, if $k \geq 0$, then

$$
\begin{aligned}
U T_{\sigma, \theta}\left(X_{k}\right)=U\left(X_{k+1}\right) & =\left(B_{\sigma, e^{i \theta}}\right)^{k+1} U\left(X_{0}\right) \\
& =B_{\sigma, e^{i \theta}}\left(B_{\sigma, e^{\imath \theta}}\right)^{k} U\left(X_{0}\right) \\
& =B_{\sigma, e^{i \theta}} U\left(X_{k}\right)
\end{aligned}
$$

and it is seen that

$$
U T_{\sigma, 0}=B_{\sigma, e^{i \theta}} U
$$

This shows that $U$ implements a unitary equivalence between $T_{\sigma, \theta}$ and $B_{\sigma, x^{2}}$ and concludes the proof of Proposition 5.2.

Proposition 5.2 motivates the following definition.

Definition 5.5. The Brownian shift of covariance $\sigma(\sigma>0)$ and angle $\theta$ is the block operator $B_{\sigma, e^{i \theta}}$ acting on $H^{2} \oplus \mathbb{C}$ defined by

$$
B_{\sigma, e^{i \theta}}=\left[\begin{array}{cc}
S & \sigma(1 \\
0 & e^{i \theta}
\end{array}\right]
$$

The Brownian shift of covariance 0 and angle $\theta$ is the operator multiplication by $e^{i \theta}$ acting on $\mathbb{C}$.

It should be clear that Brownian shifts arise naturally in the theory of non-stationary random processes. The following proposition makes it clear that Brownian shifts also arise. naturally in the theory of 2 -isometries. Recall that if $T$ is a finitely cyclic 2 -isometry, then $\Delta_{T} \geq 0$ and $\Delta_{T}$ is compact. What are the 2 -isometries with $\operatorname{rank} \Delta_{T}=1$ ? 
Proposition 5.6. Let $T \in \mathcal{L}(\mathcal{H})$ be a 2-isometry. rank $\triangle_{T}=1$ if and only if $T$ is unitarily equivalent to an operator of the form $V \oplus B_{\sigma, e^{i \theta}}$ (the $V$ summand may be absent) where $V$ is an isometry, $\sigma>0$ and $\theta \in \mathbb{R}$.

Proof. By Theorem 1.26, $T$ has the form $V \oplus T_{0}$ where $T_{0}$ is a pure 2-isometry. Note that since rank $\triangle_{T}=1$, rank $\triangle_{T_{0}}=1$. By Theorem 1.26, $T_{0}$ has the form

$$
T_{0}=\left[\begin{array}{cc}
W & f \otimes 1 \\
0 & c
\end{array}\right]
$$

acting on ker $\triangle_{T_{0}} \oplus \mathbb{C}$ where $W$ is an isometry. Also note that (1.31) in Theorem 1.26 implies that

$$
W^{*} f=0
$$

Using (5.7), we thus find

$$
\triangle_{T_{0}}=\left[\begin{array}{cc}
0 & 0 \\
0 & \|f\|^{2}+|c|^{2}-1
\end{array}\right]
$$

Since $T_{0}^{*} \triangle_{T_{0}} T_{0}=\triangle_{T_{0}}$, we deduce that

$$
\left(\|f\|^{2}+|c|^{2}-1\right)|c|^{2}=\|f\|^{2}+|c|^{2}-1 .
$$

Since rank $\triangle_{T_{0}}=1, \triangle_{T_{0}} \neq 0$ and we conclude from (5.9) that $|c|^{2}=1$. Let $c=e^{i \theta}$.

Now, note that since $f \in \operatorname{ker} W^{*}$, in fact $W$ is a pure isometry of multiplicity 1 . This is because $\bigvee\left\{W^{k} f: k \geq 0\right\}$ is reducing for $W$ and $T_{0}$ is pure.

The proof of Proposition 5.6 is now completed by observing that if $U: H^{2} \rightarrow \operatorname{ker} \triangle_{T_{0}}$ is the Hilbert space isomorphism such that $U^{*} W U$ is the unilateral shift acting on $H^{2}$ and $U 1=f /\|f\|$, then

$$
(U \oplus I)^{*} T_{0}(U \oplus I)=B_{\|f\|: e^{i \theta}}
$$

Before proceeding, let us note that there exists a pure 2 -isometry $T$ such that $\operatorname{rank} \triangle_{T}=2$ and $T$ is not a direct sum of two Brownian shifts. Indeed, if $T \in \mathcal{L}\left(H^{2} \oplus H^{2} \oplus \mathbb{C} \oplus \mathbb{C}\right)$ is given by

$$
T=\left[\begin{array}{cccc}
S & 0 & \sqrt{2} \otimes 1 & 0 \\
0 & S & 0 & \sigma \otimes 1 \\
0 & 0 & 1 & b \\
0 & 0 & 0 & -1
\end{array}\right]
$$

then $T$ is a 2 -isometry and $\operatorname{rank} \Delta_{T}=2$ if $b \neq 0$ or $\sigma \neq 0$. If $b \neq 0$, then $\operatorname{ker}\left(T^{*}-1\right)$ is not orthogonal to $\operatorname{ker}\left(T^{*}+1\right)$ and so $T$ is not a direct sum of Brownian shifts. Proposition 1.25 guarantees that $T$ is a pure 2 -isometry. 
As a final characterization of Brownian shifts we now turn to their identification in terms of analytic Dirichlet operators.

Observe that if $B_{\sigma, e^{i \theta}}$ is a Brownian shift and $\gamma=\left(\begin{array}{l}f \\ c\end{array}\right) \in H^{2} \oplus \mathbb{C}$, then by Theorem 3.23 there exist an analytic Dirichlet operator $A=P L \mid \mathcal{D}_{a}$ such that

$$
L(\varphi)(\bar{\psi})=\left\langle\varphi\left(B_{\sigma, e^{i \theta}}\right) \gamma, \psi\left(B_{\sigma, e^{i \theta}}\right) \gamma\right\rangle, \quad \psi \in \mathcal{D}_{a}
$$

To compute the slope of $L$ for this special case, simply note that

$$
\begin{aligned}
\int e^{i\left(k_{1}-k_{2}\right) \theta} d \mu & =\left(\frac{d}{d D} L\right)\left(e^{i k_{1} \theta}\right)\left(e^{-i k_{2} \theta}\right) & & \text { (Definition of slope) } \\
& =\left[\triangle_{M_{A}}\left(e^{i k_{1} \theta}\right), e^{i k_{2} \theta}\right]_{A} & & \text { (Proposition 3.13) } \\
& =\left\langle\triangle_{B_{\sigma, \theta}} B_{\sigma, e^{i \theta}}^{k_{1}} \gamma, B_{\sigma, e^{i \theta}}^{k_{2}} \gamma\right\rangle & & \text { (Definition 5.5) } \\
& =\sigma^{2}|c|^{2} e^{i\left(k_{1}-k_{2}\right) \theta}, & &
\end{aligned}
$$

so that $\mu=\sigma^{2}|c|^{2} \delta_{e^{i \theta}}$.

The previous calculation turns out to be the whole story.

Proposition 5.10. Let $T \in \mathcal{L}(\mathcal{H})$ be a nonisometric cyclic 2-isometry. $T$ is a Brownian shift if and only if for every $\gamma \in \mathcal{H}$ either

(i) the slope of $(T, \gamma)$ is a nonzero point mass or

(ii) the slope of $(T, \gamma)^{\wedge}$ is zero and $T \mid \bigvee\left\{T^{k} \gamma: k \geq 0\right\}$ is a pure cyclic isometry.

Proof. If $T$ is a Brownian shift then by the remarks preceding the proposition either (i) or (ii) holds for each $\gamma \in \mathcal{H}$ ((i) holds if $c \neq 0$ and (ii) holds if $c=0$ ). Conversely, assume that $T$ is a nonisometric cyclic 2 -isometry such that either (i) or (ii) holds for each $\gamma \in \mathcal{H}$. Fix a cyclic vector $\gamma$ for $T$. Since $T$ is nonisometric (ii) is false so (i) must hold. Let $w \delta_{p^{2}}$. be the slope of $A=(T, \gamma)^{\wedge}(w>0)$. Thus, by Proposition 3.13,

$$
\begin{aligned}
\left\langle\triangle_{T} \varphi(T) \gamma, \varphi(T) \gamma\right\rangle & =\frac{d}{d D} A(\varphi)(\bar{\varphi}) \\
& =\int \varphi \bar{\varphi}\left(w d \delta_{e^{\imath \theta}}\right) \\
& =w\left|\varphi\left(e^{i \theta}\right)\right|^{2}
\end{aligned}
$$

and it follows that

$$
\text { ker } \triangle_{T} \supseteq\left\{\varphi(T) \gamma: \varphi\left(e^{i \theta}\right)=0\right\} \text {. }
$$

But, since $\gamma$ is cyclic for $T$,

$$
\operatorname{codim}\left\{\varphi(T) \gamma: \varphi\left(e^{i \ell}\right)=0\right\} \leq 1 .
$$


Hence, rank $\triangle_{T} \leq 1$ and since $T$ is assumed non-isometric, in fact, rank $\triangle_{T}=1$. Applying Proposition 5.6 we deduce that either $T$ is a Brownian shift or $T$ has the form $V \oplus B_{\sigma, e^{i \theta}}$ for some isometry $V$. The proof of Proposition 5.10 will be complete once we have ruled out the possibility of $T$ having an isometric direct summand. We argue by contradiction. Accordingly, assume that $T \cong V \oplus B_{\sigma, \theta}$ for some isometry $V$.

We first claim that $V$ is unitary. Since $T$ is cyclic, ind $(T) \geq-1$. But

$$
\text { ind }(T)=\operatorname{ind}(V)+\operatorname{ind}\left(B_{\sigma, \theta}\right)=\operatorname{ind}(V)-1 \text {. }
$$

Hence ind $(V) \geq 0$ and $V$ is unitary. Now, since $T$ is cyclic, so also is $V$ cyclic. Choosing $f$ cyclic for $V$ and applying (ii) with $\gamma=f \oplus 0$ gives that $V$ is a pure isometry. This contradiction concludes the proof of Proposition 5.10.

In the theory of stationary stochastic processes, the one dimensional process $S_{\theta}$ with covariance furction

$$
E_{\theta}\left(X_{k_{1}} \overline{X_{k_{2}}}\right)=e^{i\left(k_{1}-k_{2}\right) \theta}
$$

plays a preminent role. The time shift operator is just the rank one unitary $\left[e^{i \theta}\right]$, and the central theorem in the subject is that every stationary process $E$ is an average of these one dimensional processes. Specifically, there exists a positive measure $\mu$ such that

$$
E\left(X_{k_{1}} \overline{X_{k_{2}}}\right)=\int e^{i\left(k_{1}-k_{2}\right) \theta} d \mu(\theta) \text {. }
$$

The underlying operator theory consists of two observations. Firstly, in the bilateral case, the spectral theorem, which asserts that every unitary is a direct integral of rank 1 unitaries (i.e. rank one opcrators $\left[e^{i \theta}\right]$ ). Secondly, in the unilateral case, the well known fact that cvery isometry has an extension to a unitary operator.

In the theory of 2-stationary processes, it turns out that processes with time shift operator $B_{\tau, e^{2}}$ play the role that rank one unitaries play in the theory of stationary processes. Thus, a basic question in the theory of 2 -stationary processes would be to identify the class of direct integrals of Brownian shifts. This class of operators would play the role for 2-stationary processes that unitary operators play in the theory of stationary processes.

Definition 5.11 A Brownian unitary of covariance 0 is a unitary operator. A Brownian unitary of covariance $\sigma>0$ is an operator which is unitarily equivalent to

$$
U \odot \int_{\oplus} B_{\sigma, e^{2 \theta}}^{(n(\theta))} d \mu(\theta)
$$

(the $U$ summand may be absent) acting on $\mathcal{H} \oplus \int_{\oplus)}\left(H^{2} \oplus \mathbb{C}\right)^{(n(\theta))} d \mu(\theta)$ where $U \in \mathcal{L}(\mathcal{H})$ is unitary, $\mu$ is a nonzero finite positive measure on $[0,2 \pi)$ and $n:[0,2 \pi) \rightarrow \mathbb{N} \cup\{\infty\}$ is a (l-measurable multiplicity function.

A characterization of Brownian unitaries can be given in terms of block operators. 
Proposition 5.12. $B$ is a Brownian unitary of covariance 0 , if and only if $B$ is unitary. $B \in \mathcal{L}(\mathcal{K})$ is a Brownian unitary of covariance $\sigma>0$ if and only if $B$ has the block matrix form

$$
B=\left[\begin{array}{cc}
V & \sigma E \\
0 & U
\end{array}\right]
$$

with respect to a decomposition $\mathcal{K}=\mathcal{K}_{1} \oplus \mathcal{K}_{2}$ where

(i) $V \in \mathcal{L}\left(\mathcal{K}_{1}\right)$ is an isometry,

(ii) $U \in \mathcal{L}\left(\mathcal{K}_{2}\right)$ is unitary, and

(iii) $E: \mathcal{K}_{2} \rightarrow \mathcal{K}_{1}$ is an isometry which maps $\mathcal{K}_{2}$ onto ker $V^{*}$.

Proof. The first assertion of the proposition comes directly from Definition 5.11.

To sce the second assertion, first assume that $B \in \mathcal{L}(\mathcal{K})$ is a Brownian unitary of covariance $\sigma>0$ so that $B$ has the form

$$
B=U_{0} \oplus \int_{\oplus} B_{\sigma, e^{i \theta}}^{(n(\theta))} d \mu(\theta)
$$

acting on $\mathcal{K}=\mathcal{K}_{0} \oplus \int_{\oplus}\left(H^{2} \oplus \mathbb{C}\right)^{(n(\theta))} d \mu(\theta)$ where $U_{0} \in \mathcal{L}\left(\mathcal{K}_{0}\right)$ is unitary, $\mu$ is a positive measure on $[0,2 \pi)$ and $n(\theta)$ is a $\mu$-measurable multiplicity function. If we define

$$
W: \mathcal{K}_{0} \oplus \int_{\oplus}\left(H^{2} \oplus \mathbb{C}\right)^{(n(\theta))} d \mu(\theta) \rightarrow\left(\mathcal{K}_{0} \oplus \int_{\oplus}\left(H^{2}\right)^{n(\theta)} d \mu(\theta)\right) \oplus \int_{\oplus} \mathbb{C}^{(n(\theta))} d \mu(\theta)
$$

by

$$
W\left(k \oplus \int_{\oplus}\left\{k_{j, \theta} \oplus c_{j, \theta}\right\}_{j=1}^{n(\theta)} d \mu(\theta)\right)=\left(k \oplus \int_{\oplus}\left\{k_{j, \theta}\right\}_{j=1}^{n(\theta)} d \mu(\theta)\right) \oplus \int_{\oplus}\left\{c_{j, \theta}\right\}_{j=1}^{n(\theta)} d \mu(\theta)
$$

then $W$ is a Hilbert space isomorphism and

$$
W B=\left[\begin{array}{ccc}
U_{0} & 0 & 0 \\
0 & V & \sigma E \\
0 & 0 & U
\end{array}\right] W
$$

where

$$
V\left(\int_{\oplus}\left\{k_{j, \theta}\right\}_{j=1}^{n(\theta)} d \mu(\theta)\right)=\int_{\oplus}\left\{S k_{j, \theta}\right\}_{j=1}^{n(\theta)} d \mu(\theta)
$$

(here, $S \in \mathcal{L}\left(H^{2}\right)$ is the unilateral shift),

$$
U\left(\int_{\oplus}\left\{c_{j, \theta}\right\}_{j=1}^{n(\theta)} d \mu(\theta)\right)=\int_{\oplus}\left\{e^{i \theta} c_{j, \theta}\right\}_{j=1}^{n(\theta)} d \mu(\theta)
$$


and

$$
E\left(\int_{\oplus}\left\{c_{j, \theta}\right\}_{j=1}^{n(\theta)} d \mu(\theta)\right)=\int_{\oplus}\left\{c_{j, \theta}\right\}_{j=1}^{n(\theta)} d \mu(\theta)
$$

Since $\left[\begin{array}{c}0 \\ E\end{array}\right]$ maps $\int_{\oplus} \mathbb{C}^{(n(\theta))} d \mu(\theta)$ onto $\operatorname{ker}\left[\begin{array}{cc}U^{*} & 0 \\ 0 & V^{*}\end{array}\right]=0 \oplus$ ker $V^{*}, B$ has the desired block form.

Now suppose that $B$ is given by (5.13) and that (i), (ii), and (iii) hold. Since $E$ maps $\mathcal{K}_{2}$ onto ker $V^{*}$, the subspace $\mathcal{M}_{1} \subseteq \mathcal{K}_{1}$ defined by

$$
\mathcal{M}_{1}=\bigvee\left\{V^{k} E \mathcal{K}_{2}: k \geq 0\right\}
$$

reduces $V$ and $V \mid \mathcal{M}_{1}^{\perp}$ is unitary. If we set $E_{0}: \mathcal{K}_{2} \rightarrow$ ker $V^{*}$ by $E_{0} k=E k$ for all $k \in \mathcal{K}_{2}$ and define the linear map

$$
W:\left(\mathcal{K}_{1} \ominus \mathcal{M}_{1}\right) \oplus H_{\mathcal{K}_{2}}^{2} \oplus \mathcal{K}_{2} \rightarrow\left(\mathcal{K}_{1} \ominus \mathcal{M}_{1}\right) \oplus \mathcal{M}_{1} \oplus \mathcal{K}_{2}
$$

densely by

$$
W(x \oplus p(\lambda) y \oplus k)=x \oplus p(V) E_{0} y \oplus k
$$

for $x \in \mathcal{K}_{1} \ominus \mathcal{M}_{1}, p$ a polynomial and $y, k \in \mathcal{K}_{2}$, then $W$ is a Hilbert space isomorphism and

$$
\left[\begin{array}{ccc}
V \mid \mathcal{K}_{1} \ominus \mathcal{M}_{1} & 0 & 0 \\
0 & V \mid \mathcal{M}_{1} & \sigma E \\
0 & 0 & U
\end{array}\right] W=W\left[\begin{array}{ccc}
V \mid \mathcal{K}_{1} \ominus \mathcal{M}_{1} & 0 & 0 \\
0 & \mathcal{M}_{\lambda} & \sigma E_{1} \\
0 & 0 & U
\end{array}\right]
$$

where $E_{1}: \mathcal{K}_{2} \rightarrow H_{\mathcal{K}_{2}}^{2}$ is the inclusion map. By the spectral theorem, there exists a positive measure $\mu$ on $\partial \mathrm{D}$ and a $\mu$-measurable multiplicity function $n(\theta)$ such that

$$
U=\int_{\bigoplus} e^{i \theta} d \mu(\theta)
$$

acting on $\int_{\oplus} \mathbb{C}^{(n(\theta))} d \mu(\theta)$. If $L: H_{\mathcal{K}_{2}}^{2} \oplus \mathcal{K}_{2} \rightarrow \int_{\oplus}\left(H^{2} \oplus \mathbb{C}\right)^{(n(\theta))} d \mu(\theta)$ is a densely defined linear map given by

$$
\begin{gathered}
L\left(p(\lambda) \int_{\oplus}\left\{c_{j, \theta}\right\}_{j=1}^{n(\theta)} d \mu(\theta) \oplus \int\left\{d_{j, \theta}\right\}_{j=1}^{n(\theta)} d \mu(\theta)\right) \\
=\int_{\oplus}\left\{p(\lambda) c_{j, \theta} \oplus d_{j, \theta}\right\}_{j=1}^{n(\theta)} d \mu(\theta)
\end{gathered}
$$

then $L$ is a Hilbert space isomorphism and

$$
L\left[\begin{array}{cc}
\mathcal{M}_{\lambda} & \sigma E_{1} \\
0 & U
\end{array}\right]=\left(\int_{\oplus} B_{\sigma, e^{i \theta}}^{(n(\theta))} d \mu(\theta)\right) L
$$


Since $E$ maps onto ker $V^{*}, V \mid \mathcal{K}_{1} \ominus \mathcal{M}_{1}$ is unitary. Thus $B$ is unitarily equivalent to

$$
B \cong\left(V \mid\left(\mathcal{K}_{1} \ominus \mathcal{M}_{1}\right)\right) \oplus \int_{\oplus} B_{\sigma, e^{i \theta}}^{(n(\theta))} d \mu(\theta)
$$

and so is a Brownian unitary of covariance $\sigma$. This establishes Proposition 5.12.

Before continuing we remark that if $B$ is a Brownian unitary of covariance $\sigma$ and if $\mathcal{M}_{1}$ is defined as in the proof of Proposition 5.12, then $B$ decomposes as

$$
B=U_{0} \oplus B_{0}
$$

with respect to $\mathcal{M}_{1} \oplus \mathcal{M}_{1}^{\perp}$ where $U_{0}$ is unitary and $B_{0}$ is a Brownian unitary of covariance $\sigma$ of the special form

$$
B_{0}=\left[\begin{array}{cc}
V & \sigma E \\
0 & U
\end{array}\right]
$$

where $V$ is a pure isometry, $U$ is unitary, and $E$ is a Hilbert space isomorphism onto $\operatorname{ker} V^{*}$. Observe that in the decomposition (5.17) $B_{0}$ is exactly the pure part of $B$ as defined in Definition 1.8. In particular, a Brownian unitary of covariance $\sigma$ is pure if and only if. it has the block form of (5.18).

We introduce the following notation for pure Brownian unitaries. If $B_{0}$ has the form given in (5.18), then it is well known that $V$ is unitarily equivalent to the operator $M_{\lambda}$ acting on $H_{\mathrm{ker} V^{*}}^{2}$. This fact together with the notation we have already introduced for Brownian shifts suggests that we define

$$
B_{\sigma E, U}=\left[\begin{array}{cc}
M_{\lambda} & \sigma E \\
0 & U
\end{array}\right]
$$

whenever $U \in \mathcal{L}(\mathcal{K})$ is a unitary operator and $E: \mathcal{K} \rightarrow \mathcal{H}$ is a Hilbert space isomorphism. In (5.19), $M_{\lambda}$ acts on $H_{\mathcal{H}}^{2}$ and $\mathcal{H}$ is regarded as a subspace of $H_{\mathcal{H}}^{2}$ in the usual way. We note that since $\operatorname{dim} \mathcal{K}=\operatorname{dim} \mathcal{H}$ one can always assume $E$ is the identity in (5.19). It turns out however to be more convenient to carry $E$ around as a marker. In any event, two pure Brownian unitaries $B_{\sigma E_{1}, U_{1}}$ and $B_{\sigma E_{2}, U_{2}}$ are unitarily equivalent if and only if $U_{1}$ and $U_{2}$ are unitarily equivalent.

Now, recall that the spectral theorem asserts that a unitary operator $U$ (defined by requiring $U U^{*}=U^{*} U=1$ ) is a direct integral of the rank one operators $\left[e^{i \theta}\right]$. Thus, the spectral theorem for unitary operators may be thought of as giving an algebraic characterization of the operators that are unitarily equivalent to direct integrals of $\left[e^{i \theta}\right]$. We now derive an analogous theorem for Brownian unitarics. 
Theorem 5.20. (Spectral Theorem for Brownian unitaries) Let $T \in \mathcal{L}(\mathcal{H}), \triangle=$ $T^{*} T-I$, and $\sigma=\|\triangle\|^{\frac{1}{2}}$. Suppose that $\sigma>0$. $T \in \mathcal{L}(\mathcal{H})$ is a Brownian unitary if and only if

(i) $T^{* 2} T^{2}-2 T^{*} T+I=0$,

(ii) $\triangle\left(T T^{*}-I\right) \triangle=0$,

(iii) $\sigma^{-2} \triangle$ is a projection, and

(iv) there exists a projection $Q \in \mathcal{L}(\mathcal{H})$ such that $\left(\sigma^{2}-\triangle\right)\left(T T^{*}-I\right)\left(\sigma^{2}-\triangle\right)=$ $\sigma^{4}\left(\sigma^{2}-1\right) Q$.

Proof. To see that (i)-(iv) hold if $T$ is a Brownian unitary, first observe that if $T_{\theta}$ satisfy (i)-(iv), then $\int_{\oplus} T_{\theta} d \mu(\theta)$ satisfies (i)-(iv). Therefore, it suffices to show every unitary and every Brownian shift of covariance $\sigma$ satisfies (i)-(iv). This follows directly from computation.

Suppose now that (i)-(iv) hold. Since $\sigma>0, \triangle \neq 0$ and so ran $\triangle \neq\{0\}$. We claim that $\operatorname{ran} \triangle \neq \mathcal{H}$. For if $\operatorname{ran} \triangle=\mathcal{H}$, then $T^{*} T=1+\sigma^{2}$. Consequently, $T=\sqrt{1+\sigma^{2}} V$ for some isometry $V$, which would violate (i) since $\sigma>0$. Summarizing, we have shown that if $\mathcal{H}_{1}=$ ker $\triangle$ and $\mathcal{H}_{2}=(\operatorname{ran} \Delta)^{-}$, then $\mathcal{H}=\mathcal{H}_{1} \oplus \mathcal{H}_{2}$ is a nontrivial decomposition of $\mathcal{H}$.

Now, by Theorem 1.26, $T$ has the block matrix form

$$
T=\left[\begin{array}{cc}
V & \sigma E \\
0 & X
\end{array}\right]
$$

with respect to the decomposition $\mathcal{H}=\mathcal{H}_{1} \oplus \mathcal{H}_{2}$ where

$$
\begin{gathered}
V \in \mathcal{L}\left(\mathcal{H}_{1}\right) \quad \text { is an isometry, } \\
\sigma^{2} E^{*} E+X^{*} X-I \text { is injective, } \\
X^{*}\left(\sigma^{2} E^{*} E+X^{*} X-I\right) X=\sigma^{2} E^{*} E+X^{*} X-I,
\end{gathered}
$$

and

$$
E \text { maps } \mathcal{H}_{2} \text { densely into } \operatorname{ker} V^{*} \text {. }
$$

Using (5.21) one obtains the formula

$$
\Delta=\left[\begin{array}{cc}
0 & 0 \\
0 & \sigma^{2} E^{*} E+X^{*} X-1
\end{array}\right] .
$$

Thus, (iii) and (5.23) imply that

$$
\sigma^{2} E^{*} E+X^{*} X-I=\sigma^{2} .
$$


Now, (5.24) and (5.27) imply that $X$ is an isometry and so by another application of (5.27), $E$ is an isometry. In particular, note that (5.26) becomes

$$
\triangle=\left[\begin{array}{cc}
0 & 0 \\
0 & \sigma^{2}
\end{array}\right]
$$

We claim that in fact $X$ is unitary. Substituting (5.21) and (5.28) into (ii) gives the equation

$$
\sigma^{4}\left(X X^{*}-1\right)=0
$$

Accordingly, $X X^{*}=1$, and $X$ is unitary. Also, observe that

$$
T T^{*}-1=\left[\begin{array}{cc}
V V^{*}+\sigma^{2} E E^{*}-1 & \sigma E X^{*} \\
\sigma X E^{*} & 0
\end{array}\right]
$$

Now, substituting (5.28) and (5.29) into (iv) yields the equation

$$
\sigma^{4}\left[\begin{array}{cc}
V V^{*}+\sigma^{2} E E^{*}-1 & 0 \\
0 & 0
\end{array}\right]=\sigma^{4}\left(\sigma^{2}-1\right) Q
$$

We claim that (5.30) implies that

$$
V V^{*}+\sigma^{2} E E^{*} \quad \text { is invertible. }
$$

If $\sigma=1$, then (5.30) implics that

$$
V V^{*}+\sigma E E^{*}=1
$$

so that (5.31) holds. If $\sigma \neq 1$, then (5.30) implies that $Q$ has the form

$$
Q=\left[\begin{array}{cc}
Q_{0} & 0 \\
0 & 0
\end{array}\right]
$$

where necessarily $Q_{0}$ is a projection. In particular, we obtain the equation,

$$
V V^{*}+\sigma^{2} E E^{*}=\left(1-Q_{0}\right)+\sigma^{2} Q_{0},
$$

and again we see that (5.31) holds.

Summarizing we have shown that $T$ has the form given in (5.21) where $V$ is an isometry, $X$ is unitary, $E$ is an isometry whose range is dense in ker $V^{*}$ (and hence equals ker $V^{*}$ ), and $V^{*} E=0$. Hence $T$ is a Brownian unitary and the proof of Theorem 5.20 is complete.

Theorem 5.20 has the following immediate corollary. 
Lemma 5.32. If $B \in \mathcal{L}(\mathcal{H})$ is a Brownian unitary of covariance $\sigma$ and $\mathcal{M} \subseteq \mathcal{H}$ is a reducing subspace for $B$, then either $B \mid \mathcal{M}$ is unitary or $B \mid \mathcal{M}$ is a Brownian unitary of covariance $\sigma$.

Before giving the algebraic proof of the lifting theorem (Theorem 5.80 below), we present another class of 2-isometries which we shall call Brownian isometries. This class of 2-isometries arises naturally not only in the analytic proof of the lifting theorem given in Section 6 (Theorem 6.20) but also in the theory of minimal lifts considered in Section 9 .

Note that in the definition of Brownian unitaries the direct integrals were taken over Brownian shifts of a constant covariance $\sigma$. We now consider direct integrals where we vary both the covariance and angle of the Brownian shifts.

Definition 5.33. A Brownian isometry of covariance 0 is an isometry. A Brownian isometry of covariance $\sigma>0$ is an operator $B$ such that $\sigma^{2}=\left\|B^{*} B-I\right\|$ and which is unitarily equivalent to

$$
V_{0} \oplus \int_{\oplus} B_{t E_{t}, U_{t}} d \mu(t)
$$

(the $V_{0}$ summand may be absent) acting on $\mathcal{H} \oplus \int_{\oplus} H_{\mathcal{K}_{t}}^{2} \oplus \mathcal{K}_{t} d \mu(t)$ where $V_{0} \in \mathcal{L}(\mathcal{H})$ is an isometry, $\mu$ is a nonzero positive measure on $(0, \sigma], U_{t} \in \mathcal{L}\left(\mathcal{K}_{t}\right)$ is unitary for $\mu$-a.e. $t$,

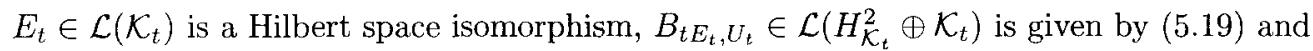
the following two measurability conditions are satisfied:

$$
t \mapsto \operatorname{dim} \mathcal{K}_{t} \quad \text { is a } \mu \text {-measurable function }
$$

$$
\text { If } X_{n}=\left\{t \in(0, \sigma]: \operatorname{dim} \mathcal{K}_{t}=n\right\}, \mu_{n}(E)=\mu\left(E \cap X_{n}\right)
$$
for all measurable $E$ and for all $n \in \mathbb{N} \cup\{\infty\}$, and $\mathcal{K}_{t_{1}}$ and $\mathcal{K}_{t_{2}}$ are identified for $t_{1}, t_{2} \in X_{n}$, then the maps on $X_{n}$ given by $t \mapsto E_{t}$ and $t \mapsto U_{t}$ are $\mu_{n}$ measurable. (Recall that $t \mapsto E_{t}$ is measurable if and only if $t \mapsto\left\langle E_{t} x, y\right\rangle$ is measurable for all $x$ and $y$.)

As in the case of Brownian unitaries, Brownian isometries can both be expressed in the form of block operators (Proposition 5.12) as well as characterized algebraically (Theorem $5.20)$.

Proposition 5.37. $B$ is a Brownian isometry of covariance 0 if and only if $B$ is an isometry. $B \in \mathcal{L}(\mathcal{K})$ is a Brownian isometry of covariance $\sigma>0$ if and only if $\sigma^{2}=$ $\left\|B^{*} B-I\right\|$ and $B$ has the block matrix form

$$
B=\left[\begin{array}{cc}
V & \sigma E \\
0 & U
\end{array}\right]
$$


with respect to a decomposition $\mathcal{K}=\mathcal{K}_{1} \oplus \mathcal{K}_{2}$ where

(i) $V \in \mathcal{L}\left(\mathcal{K}_{1}\right)$ is an isometry,

(ii) $U \in \mathcal{L}\left(\mathcal{K}_{2}\right)$ is unitary ,

(iii) $E: \mathcal{K}_{2} \rightarrow \mathcal{K}_{1}$ is an injective contraction which maps $\mathcal{K}_{2}$ into ker $V^{*}$, and

(iv) $E^{*} E$ commutes with $U$.

Proof. The first assertion of the proposition follows directly from Definition 5.33.

To see the second assertion, first assume that $B$ is the Brownian isometry of (5.34). If the operators $V, E$, and $U$ are defined by the formulas,

$$
\begin{aligned}
U & =\int_{\oplus} U_{t} d \mu(t), \\
E & =\frac{1}{\sigma} \int_{\oplus} t E_{t} d \mu(t), \quad \text { and } \\
V & =V_{0} \oplus \int_{\oplus} M_{\lambda} d \mu(t),
\end{aligned}
$$

then (i)-(iv) are obtained and $B$ has the desired block form.

Now suppose that $B$ has the form given in (5.38) and that (i)-(iv) hold. If we set

$$
\mathcal{M}_{1}=\bigvee\left\{V^{k} E \mathcal{K}_{2}: k \geq 0\right\}
$$

then, since $V^{*} E=0, \mathcal{M}_{1}$ reduces $V$. Thus $B$ decomposes as

$$
B=\left[\begin{array}{ccc}
V \mid \mathcal{K}_{1} \ominus \mathcal{M}_{1} & 0 & 0 \\
0 & V \mid \mathcal{M}_{1} & \sigma E \\
0 & 0 & U
\end{array}\right]
$$

acting on $\mathcal{K}=\left(\mathcal{K}_{1} \oplus \mathcal{M}_{1}\right) \oplus \mathcal{M}_{1} \oplus \mathcal{K}_{2}$. Furthermore, by construction ran $E$ is dense in $\operatorname{ker}\left(V \mid \mathcal{M}_{1}\right)^{*}$. Since $E$ is injective it follows that there exists a Hilbert space isomorphism $L: \mathcal{K}_{2} \rightarrow \operatorname{ker}\left(V \mid \mathcal{M}_{1}\right)^{*}$ and a positive $P \in \mathcal{L}\left(\mathcal{K}_{2}\right)$ such that $E=L P$. Consequently, $B$ is unitarily equivalent to an operator of the form

$$
\left[\begin{array}{ccc}
V_{0} & 0 & 0 \\
0 & M_{\lambda} & \sigma P \\
0 & 0 & U
\end{array}\right]
$$

acting on $\mathcal{K}_{1} \ominus \mathcal{M}_{1} \oplus H_{\mathcal{K}_{2}}^{2} \oplus \mathcal{K}_{2}$. Here $V_{0}=V \mid \mathcal{K}_{1} \ominus \mathcal{M}_{1}$ is an isometry. To express (5.40) in the form (5.34) first observe that since $E^{*} E$ commutes with $U$, so also $P=\left(E^{*} E\right)^{\frac{1}{2}}$ commutes with $U$. Consequently, if we express

$$
P=\int_{\ominus} t d \mu(t)
$$


acting on

$$
\mathcal{K}_{2}=\int_{\oplus} \mathcal{H}_{t} d \mu(t)
$$

then $U$ has the form

$$
U=\int_{\oplus} U_{t} d \mu(t)
$$

Furthermore, since (5.42) gives a decomposition of the initial space of $M_{\lambda}, M_{\lambda}$ decomposes

$$
M_{\lambda}=\int_{\oplus} M_{\lambda} d \mu(t)
$$

Combining (5.40)-(5.44) gives the desired resolution of $B$ as a direct integral and concludes the proof of Proposition 5.37.

Before continuing we remark that if $B$ is a Brownian isometry of covariance $\sigma$ and if $\mathcal{M}_{1}$ is defined as in the proof of Proposition 5.37, then $B$ decomposes as

$$
B=V_{0} \oplus B_{0}
$$

with respect to $\mathcal{M}_{1} \oplus \mathcal{M}_{1}^{\perp}$ where $V_{0}$ is an isometry and $B_{0}$ is a Brownian isometry of covariance $\sigma$ of the special form

$$
B_{0}=\left[\begin{array}{cc}
V & \sigma E \\
0 & U
\end{array}\right]
$$

where $V$ is a pure isometry, $U$ is unitary and $E$ is an injective contraction with ( $\operatorname{ran} E)^{-}=$ ker $V^{*}$. Observe that in the decomposition (5.45) $B_{0}$ is exactly the pure part of $B$ as defined in Definition 1.8. In particular, a Brownian isometry of covariance $\sigma$ is pure if and only if it has the block form of (5.46).

We introduce the following notation for pure Brownian isometries. If $B_{0}$ has the form given in (5.46), then it is well known that $V$ is unitarily equivalent to the operator $\mathcal{M}_{\lambda}$ acting on $H_{\text {ker } V^{*}}^{2}$. This fact together with the notation we have already introduced for Brownian unitaries suggest that we define

$$
B_{\sigma E, U}=\left[\begin{array}{cc}
\mathcal{M}_{\lambda} & \sigma E \\
0 & U
\end{array}\right]
$$

whenever $U \in \mathcal{L}(\mathcal{K})$ is a unitary operator, $E: \mathcal{K} \rightarrow \mathcal{H}$ is an injective contraction with dense range and $E^{*} E$ commutes with $U$. In (5.47), $\mathcal{M}_{\lambda}$ acts on $H_{\mathcal{H}}^{2}$ and $\mathcal{H}$ is regarded as a subspace of $H_{\mathcal{H}}^{2}$ in the usual way. 
Theorem 5.48. (Spectral Theorem for Brownian isometries) Let $T \in \mathcal{L}(\mathcal{H})$ and $\triangle=$ $T^{*} T-I . T$ is a Brownian isometry if and only if

(i) $T^{* 2} T^{2}-2 T^{*} T+I=0$

(ii) $\triangle\left(T T^{*}-I\right) \triangle=0$

Proof. As in the proof of Proposition 5.12, to prove that (i) and (ii) hold for any Brownian isometry, it suffices to show that (i) and (ii) hold for every isometry and every Brownian shift, facts that follow directly from computation.

Suppose now that (i) and (ii) hold. Set $\sigma=\|\triangle\|^{\frac{1}{2}}$. If $\sigma=0$, then $T$ is an isometry and so $T$ is a Brownian isometry. Thus assume $\sigma>0$. As in the proof of Theorem 5.20 if $\mathcal{H}_{1}=$ ker $\triangle$ and $\mathcal{H}_{2}=(\operatorname{ran} \triangle)^{-}$, then $\mathcal{H}=\mathcal{H}_{1} \oplus \mathcal{H}_{2}$ is a nontrivial decomposition of $\mathcal{H}$ and with respect to this decomposition

$$
T=\left[\begin{array}{cc}
V & \sigma E \\
0 & X
\end{array}\right]
$$

where

$$
V \in \mathcal{L}\left(\mathcal{H}_{1}\right) \text { is an isometry, }
$$

and

$$
E \quad \text { maps } \mathcal{H}_{2} \text { into } \operatorname{ker} V^{*} \text {. }
$$

Now, by Proposition 5.37, $T$ will be a Brownian isometry provided $X$ is unitary, $E$ is an injective contraction and $E^{*} E$ commutes with $X$.

To see that $X$ is unitary observe that (5.49) implies the formula

$$
\triangle=\left[\begin{array}{cc}
0 & 0 \\
0 & \sigma^{2} E^{*} E+X^{*} X-I
\end{array}\right] .
$$

Substituting (5.54) into (ii) yields

$$
\left(\sigma^{2} E^{*} E+X^{*} X-I\right)\left(X X^{*}-I\right)\left(\sigma^{2} E^{*} E+X^{*} X-I\right)=0 .
$$

Since $\sigma^{2} E^{*} E+X^{*} X-I$ is injective, (5.52) implies that $X$ is injective and (5.55) implies that $X$ is a co-isometry. Thus $X$ is an injective co-isometry, i.e. a unitary. 
To see that $E$ is an injective contraction observe that since $X$ is unitary, (5.54) becomes

$$
\triangle=\left[\begin{array}{cc}
0 & 0 \\
0 & \sigma^{2} E^{*} E
\end{array}\right]
$$

acting on $\operatorname{ker} \triangle \oplus(\operatorname{ran} \triangle)^{-}$and that by definition $\sigma^{2}=\|\Delta\|$.

Finally, to see that $E^{*} E$ commutes with $X$ note that since $X$ is unitary, $(5.52)$ becomes

$$
\sigma^{2} X^{*} E^{*} E X=\sigma^{2} E^{*} E
$$

Multiplying (5.56) on the left by $\sigma^{-2} X$ yields

$$
E^{*} E X=X E^{*} E
$$

and so $X$ commutes with $E^{*} E$. Thus, $T$ is a Brownian isometry, and the proof of Theorem 5.48 is complete.

Note that Theorem 5.48 has the following immediate corollary.

Corollary 5.57. If $B \in \mathcal{L}(\mathcal{H})$ is a Brownian isometry of covariance $\sigma$ and $\mathcal{M} \subseteq \mathcal{H}$ is a reducing subspace for $B$, then $B \mid \mathcal{M}$ is a Brownian isometry of covariance $\leq \sigma$.

The qualitative difference between Corollary 5.57 and Lemma 5.32 is whether the covariance can decrease or not.

Before giving the algebraic proof of the Lifting theorem (Theorem 5.80), we show that every Brownian isometry of covariance $\sigma$ lifts to a Brownian unitary of covariance $\sigma$ (Proposition 5.79). In section 7, we shall use Proposition 5.79 to complete the analytic proof of Theorem 5.80 .

Our first result which describes the invariant subspaces of Brownian shifts is a reformulation of 'Theorem 7 in [Sa]. Observe that if $\mathcal{M}$ is an invariant subspace for $B_{\sigma, e^{i \theta}}$, then $\mathcal{M}$ must be of one of the following two types. Let us agree to say that $\mathcal{M}$ is of type $I$ if

$$
\mathcal{M} \subseteq H^{2} \oplus\{0\}
$$

Otherwise, there exists a vector $g \in H^{2}$ such that

$$
\mathcal{M}=\mathbb{C}\left[\begin{array}{l}
g \\
1
\end{array}\right]+\mathcal{M}_{0}
$$

where

$$
\mathcal{M}_{0}=\left(H^{2} \oplus\{0\}\right) \cap \mathcal{M}
$$


and we shall say that $\mathcal{M}$ is of type II. By Beurling's theorem [B] it should be clear that to each invariant subspace of type I there exists a unique (up to scalar multiple) inner function $\varphi$ such

$$
\mathcal{M}=\varphi H^{2} \oplus\{0\}
$$

and conversely any inner function gives rise via (5.60) to an invariant subspace of type I.

To understand invariant subspaces of type II our first observation is that in formula (5.58) $g$ is uniquely determined by $\mathcal{M}$ provided we require that

$$
\left[\begin{array}{l}
g \\
1
\end{array}\right] \perp \mathcal{M} \cap\left(H^{2} \oplus\{0\}\right), .
$$

According, if $\mathcal{M}$ is an invariant subspace of type II, we define $g_{\mathcal{M}}$ to be the vector in $H^{2}$ satisfying (5.58), (5.59) and (5.61). In addition we can attach to any invariant subspace $\mathcal{M}$ of type II the inner function $\varphi_{\mathcal{M}}$ such that

$$
\mathcal{M}_{0}=\varphi_{\mathcal{M}} H^{2} \oplus\{0\}
$$

It should be clear that the issue of describing the invariant subspace lattice of $B_{\sigma, e^{i \theta}}$ in function theoretic terms is precisely to describe which pairs $\left(g_{\mathcal{M}}, \varphi_{\mathcal{M}}\right)$ arise from invariant subspaces $\mathcal{M}$ of type II.

Proposition 5.63. If $\sigma>0, \theta \in[0,2 \pi)$ and $\mathcal{M}$ is an invariant subspace for $B_{\sigma, e^{i \theta}}$ of type $I I$, then $\varphi_{\mathcal{M}}$ has a radial limit at $e^{i \theta}$ and

$$
g_{\mathcal{M}}=\sigma \overline{\varphi_{\mathcal{M}}\left(e^{i \theta}\right)} \frac{\varphi_{\mathcal{M}}-\varphi_{\mathcal{M}}\left(e^{i \theta}\right)}{z-e^{i \theta}} .
$$

Conversely, if $\varphi$ is an inner function such $\varphi$ has radial limit $\varphi\left(e^{i \theta}\right)$ at $e^{i \theta}$ and

$$
\frac{\varphi-\varphi\left(e^{i \theta}\right)}{z-e^{i \theta}} \in H^{2}
$$

then there exists a unique invariant subspace $\mathcal{M}$ of type II for $B_{\sigma, e^{i \theta}}$ such that $\varphi_{\mathcal{M}}=\varphi$.

Proof. First assume that $\mathcal{M}$ is an invariant subspace of type II for $B_{\sigma, e^{i \theta}}$. Let $g=g_{\mathcal{M}}$ and $\varphi=\varphi_{\mathcal{M}}$. Since

$$
B_{\sigma, e^{i \theta}}\left[\begin{array}{l}
g \\
1
\end{array}\right]=\left[\begin{array}{c}
z g+\sigma \\
e^{i \theta}
\end{array}\right]=e^{i \theta}\left[\begin{array}{l}
g \\
1
\end{array}\right]+\left[\begin{array}{c}
\left(z-e^{i \theta}\right) g+\sigma \\
0
\end{array}\right] \in \mathcal{M}
$$

(5.62) implies that

$$
\left(z-e^{i \theta}\right) g+\sigma \in \varphi H^{2} .
$$


Furthermore, (5.61) implies that

$$
g \perp \varphi H^{2}
$$

Now, (5.66) and (5.67) imply that

$$
\int z^{n}\left(\left(z-e^{i \theta}\right) g+\sigma\right) \bar{g} \frac{d t}{2 \pi}=0 \quad \text { for } \quad n \geq 0 .
$$

Hence there exists $h \in H^{1}$ such that

$$
\left(\left(z-e^{i \theta}\right) g+\sigma\right) \bar{g}=z h \quad \text { for a.e. } \quad z \in \partial \mathbb{D}
$$

Taking conjugates in (5.68) and using the identity $\overline{z-e^{i \theta}}=-\overline{e^{i \theta} z}\left(z-e^{i \theta}\right)$, yields the formula

$$
\sigma e^{i \theta} z g+\sigma \bar{g}=z h+e^{i \theta} \bar{h} \quad \text { for a.e. } z \in \partial \mathbb{D} .
$$

Hence we deduce that

$$
h=\sigma e^{i \theta} g
$$

which via (5.68) implies that in fact

$$
\left(\left(z-e^{i \theta}\right) g+\sigma\right) \bar{g}=\sigma e^{i \theta} z g
$$

Taking absolute values in (5.69) and using (5.66) yields an inner function $\psi$ such that

$$
\left(z-e^{i \theta}\right) g+\sigma=\sigma \varphi \psi
$$

Now we claim that $\psi$ is constant. Noting that (5.70) implies that

$$
g=\sigma \frac{\varphi \psi-1}{z-e^{i \theta}}
$$

we calculate using (5.67) and the identity

$$
\frac{z-e^{i \theta}}{\overline{z-e^{i \theta}}}=-e^{i \theta} z \quad, \quad z, e^{i t} \in \partial \mathbb{D}
$$

that if $n \geq 0$, then

$$
\begin{aligned}
0 & =\left\langle\left(z-e^{i \theta}\right) z^{n} \varphi, g\right\rangle \\
& =\sigma \int z^{n} \frac{z-e^{i \theta}}{\overline{z-e^{i \theta}}} \varphi(\overline{\varphi \psi-1}) \frac{d t}{2 \pi} \\
& =-e^{i \theta} \sigma \int z^{n+1}(\bar{\psi}-\varphi) \frac{d t}{2 \pi} \\
& =-e^{i \theta} \sigma \int z^{n+1} \bar{\psi} \frac{d t}{2 \pi} .
\end{aligned}
$$


Hence $\psi$ is constant.

The first assertions of Proposition 5.63 now follow by observing that (5.70) implies both that the radial limit of $\varphi$ at $e^{i \theta}$ exists $\left(\varphi\left(e^{i \theta}\right)=\bar{\psi}\right)$ and also that formula (5.64) holds.

Now assume that $\varphi$ is an inner function that has radial limit $\varphi\left(e^{i \theta}\right)$ at $e^{i \theta}$ and that (5.65) holds. Let

$$
g=\sigma \varphi\left(\overline{e^{i \theta}}\right) \frac{\varphi-\varphi\left(e^{i \theta}\right)}{z-e^{i \theta}}
$$

and note that if $\mathcal{M}$ is defined by

$$
\mathcal{M}=\mathbb{C}\left[\begin{array}{l}
g \\
1
\end{array}\right] \vee\left(\varphi H^{2} \oplus\{0\}\right)
$$

then $\mathcal{M}$ is an invariant subspace for $B_{\sigma, e^{i \theta}}$ of type II. Furthermore, by construction $\varphi_{\mathcal{M}}=$ $\varphi$. To see that $\mathcal{M}$ is unique note that if $\mathcal{M}_{1}$ is any invariant subspace of type II such that $\varphi_{\mathcal{M}_{1}}=\varphi$, then (5.64) implies that $g_{\mathcal{M}_{1}}=g$ so that $\mathcal{M}_{1}=\mathcal{M}$. This concludes the proof of Proposition 5.63.

Lemma 5.71. If $\sigma>0, \theta \in[0,2 \pi), \mathcal{N}$ is an invariant subspace for $B_{\sigma, e^{i \theta}}$ and $\mathcal{M}$ is an invariant subspace for $B_{\sigma, e^{i \theta}}$ of type $I I$, then

$$
\mathcal{M} \subseteq \mathcal{N}
$$

if and only if

$$
g_{\mathcal{M}} \oplus 1 \in \mathcal{N}
$$

Proof. Since by construction $g_{\mathcal{M}} \oplus 1$ is an element of $\mathcal{M}$ it should be clear that (5.72) implies (5.73). Conversely suppose that (5.73) holds. By (5.58) and (5.62)

$$
\mathcal{M}=\mathbb{C}\left(g_{\mathcal{M}} \oplus 1\right)+\left(\varphi_{\mathcal{M}} H^{2} \oplus\{0\}\right) .
$$

Thus, it suffices to show that $\varphi_{\mathcal{M}} \oplus 0 \in \mathcal{N}$. But, by (5.64),

$$
\begin{aligned}
\overline{\sigma_{\mathcal{M}}\left(e^{i \theta}\right)} \varphi_{\mathcal{M}} \oplus 0 & =\left(\left(z-e^{i \theta}\right) g_{\mathcal{M}}+\sigma\right) \oplus 0 \\
& =\left(B_{\sigma, \theta}-e^{i \theta}\right)\left(g_{\mathcal{M}} \oplus 1\right) \in \mathcal{N} .
\end{aligned}
$$

This establishes Lemma 5.71.

The preceeding lemma in addition to identifying the order structure for Lat $\left(B_{\sigma, e^{2 \theta}}\right)$ immediately implies the following result. 
Corollary 5.74. If $\sigma>0, \theta \in[0,2 \pi)$, and $\mathcal{M}$ is an invariant subspace for $B_{\sigma, e^{i \theta}}$ of type $I I$, then $g_{\mathcal{M}} \oplus 1$ is cyclic for $B_{\sigma, e^{i \theta}} \mid \mathcal{M}$.

By Proposition 5.6 if $B_{\sigma, e^{i \theta}}$ is a Brownian shift and $\mathcal{M}$ is an invariant subspace for $B_{\sigma, e^{i \theta}}$ of type II, then a priori

$$
\operatorname{dim} \operatorname{ran} \triangle_{B_{\sigma, e^{i \theta}} \mid \mathcal{M}}=1 .
$$

The following proposition identifies the space $\operatorname{ran} \triangle_{B_{\sigma, e^{i \theta}} \mid \mathcal{M}}$.

Proposition 5.75. If $\sigma>0, \theta \in[0,2 \pi)$ and $\mathcal{M}$ is an invariant subspace for $B_{\sigma, e^{i \theta}}$ of type II, then

$$
\operatorname{ran} \triangle_{B_{\sigma, e^{i \theta}} \mid \mathcal{M}}=\mathbb{C}\left(g_{\mathcal{M}} \oplus 1\right) \text {. }
$$

Furthermore, $B_{\sigma, e^{i \theta}} \mid \mathcal{M}$ is a Brownian shift of covariance,

$$
\frac{\sigma}{\left(1+\left\|g_{\mathcal{M}}\right\|^{2}\right)^{\frac{1}{2}}}
$$

and of angle $\theta$.

Proof. Let $\nu=\left(1+\left\|g_{\mathcal{M}}\right\|^{2}\right)^{\frac{1}{2}}$ and note by definitions of $g_{\mathcal{M}}$ and $\varphi_{\mathcal{M}}$ that

$$
\frac{g_{\mathcal{M}} \oplus 1}{\nu}, \quad \varphi_{\mathcal{M}} \oplus 0, \quad z \varphi_{\mathcal{M}} \oplus 0, \ldots
$$

is an orthonormal basis for $\mathcal{M}$. Since

$$
\begin{aligned}
\triangle_{B_{\sigma, e^{i \theta}}} & =\sigma^{2}(0 \oplus 1) \otimes(0 \oplus 1), \\
\triangle_{B_{\sigma, e^{i \theta}} \mid \mathcal{M}} & =\sigma^{2} P_{\mathcal{M}}(0 \oplus 1) \oplus P_{\mathcal{M}}(0 \oplus 1) \\
& =\frac{\sigma^{2}}{\nu^{2}} \frac{g_{\mathcal{M}} \oplus 1}{\nu} \otimes \frac{g_{\mathcal{M}} \oplus 1}{\nu} .
\end{aligned}
$$

Hence, (5.76) holds and $B_{\sigma, e^{i \theta}} \mid \mathcal{M}$ is a 2-isometry with covariance given by (5.77). Now, by Proposition 1.25 and Corollary $5.74, B_{\sigma, e^{i \theta}} \mid \mathcal{M}$ is a pure 2-isometry. Since rank $\triangle_{B \mid \mathcal{M}}=1$, Proposition 5.6 implies that $B_{\sigma, e^{i \theta}} \mid \mathcal{M}$ is a Brownian shift.

To see that $\theta$ gives the angle of $B_{\sigma, e^{i \theta}} \mid \mathcal{M}$ simply observe that if $B$ is any Brownian shift and $\gamma$ is a unit vector chosen in $\operatorname{ran} \triangle_{B}$, then the angle of $B$ is given by the formula

$$
e^{i \theta}=\langle B \gamma, \gamma\rangle
$$

Hence, since

$$
\begin{aligned}
\left\langle\left(B_{\sigma, e^{i \theta}} \mid \mathcal{M}\right) \frac{g_{\mathcal{M}} \oplus 1}{\nu}, \frac{g_{\mathcal{M}} \oplus 1}{\nu}\right\rangle & =\left\langle B_{\sigma, e^{i \theta}} \frac{g_{\mathcal{M}} \oplus 1}{\nu}, \frac{g_{\mathcal{M}} \oplus 1}{\nu}\right\rangle \\
& =e^{i \theta}
\end{aligned}
$$


the angle of $B_{\sigma, e^{i 0}} \mid \mathcal{M}$ is $\theta$ and the proof of Proposition 5.75 is complete.

We remark before continuing that if $B$ is a Brownian shift, then

$\operatorname{dim} \operatorname{ker}\left(\left\|\triangle_{B}\right\|-\triangle_{B}\right)=1$ and Corollary 5.74 implies that if $\gamma$ is a unit vector in ker $\left(\left\|\Delta_{B}\right\|-\Delta_{B}\right)$, then $\gamma$ is cyclic for $B$. In Section 8 we shall prove these facts hold for an arbitrary cyclic pure 2-isometry (Theorem 8.19).

The man application of the previous foray inte the function theory of Brownian shifts will be via Lemma 5.78 below. Fix $\sigma>0, \theta \in[0,2 \pi)$ and $\alpha \in \mathbb{D}$. If $B_{\alpha}$ is defined by

$$
B_{\alpha}(z)=\frac{z-\alpha}{1-\alpha z}
$$

then note that by Proposition 5.63 an invariant subspace $\mathcal{M}_{\alpha}$ of type II can be defined for $B_{\sigma, r^{\prime \prime \prime}}$ that satisfies the condition

$$
\varphi_{\mathcal{M}_{\mathrm{r}}}=B_{\mathrm{\alpha}}
$$

Furthermore (5.64) implies that

$$
g_{\mathcal{M}}=\sigma e^{-i \theta} \frac{\left(1-|\alpha|^{2}\right)^{\frac{1}{2}}}{1-\alpha e^{-i \theta}} \frac{\left(1-|\alpha|^{2}\right)^{\frac{1}{2}}}{1-\bar{\alpha} z}
$$

Noting that

$$
\left\|g_{\mathcal{M}_{r}}\right\|^{2}=\sigma^{2} \frac{1-|\alpha|^{2}}{\left|1-\bar{\alpha} e^{i \theta}\right|^{2}}
$$

one: sees that

$$
\left\{\frac{\sigma}{\left(1+\left\|g_{\mathcal{M}_{i k}}\right\|^{2}\right)^{\frac{1}{2}}}: \sigma \in \mathbb{D}\right\}=(0, \sigma) .
$$

Thus, by an application of Proposition 5.75 one obtains the following lemma.

Lemma 5.78. If $0 \leq \sigma_{0} \leq \sigma$ and $\theta \in[0,2 \pi)$, there exists an isometry $L \in \mathcal{L}\left(H^{2}(\mathrm{f}) \mathbb{C}\right)$ sich that.

$$
L B_{\sigma_{0}, e^{i \theta}}=B_{\sigma, e^{i \theta}} L
$$

Recalling that Brownian isometries (resp., unitaries) of covariance $\sigma$ are defined as direct integrals of Brownian shifts of covariance less than or equal to (resp,. equal to) $\sigma$ it shomld be clear that Lemmia 5.78 and the thenry of direct integrals implies the following result.

Proposition 5.79. If $B_{0} \in \mathcal{L}(\mathcal{H})$ is a Brownian isometry of covariance $\sigma_{0}$ and $\sigma_{0} \leq \sigma$, then there sxists a Hilbert space $K$, a Brownin mitary of covariance $\sigma$ and an isometry $L: \mathcal{H} \rightarrow K:$ shch that

$$
L B_{0}=B L
$$


We now show that Proposition 5.79 holds not just for the general Brownian isometry of covariance $\leq \sigma$ but in fact for the general 2-isometry of covariance $\leq \sigma$, a result which shows that Brownian unitaries play the role in the theory of 2 -isometries that unitaries play in the theory of isometries.

Theorem 5.80. If $T$ is a 2-isometry of covariance $\sigma$, then $T$ has an extension to a Brownian unitary of covariance $\sigma$.

Proof. We will attach to the 2-isometry $T$ a certain isometry $V$ and a certain contraction $C$. The Brownian unitary extension of $T$ will be constructed from a unitary extension $U$ of $V$ and an isometric dilation $S$ of $C^{*}$.

Let $T \in \mathcal{L}(\mathcal{H})$, set

$$
\delta=\left(1-\frac{1}{\sigma^{2}} \Delta_{T}\right)^{\frac{1}{2}}
$$

and let

$$
\mathcal{H}_{0}=(\operatorname{ran} \delta)^{-}
$$

Define $C_{0}:(\operatorname{ran} \delta T)+\left(\mathcal{H}_{0} \ominus(\operatorname{ran} \delta T)\right) \rightarrow \mathcal{H}_{0}$ by

$$
C_{0}(\delta T x)=\delta x, \quad x \in \mathcal{H}
$$

and

$$
C_{0} \mid \mathcal{H}_{0} \ominus(\operatorname{ran} \delta T)^{-}=0
$$

To see that $C_{0}$ is well defined and extends by continuity to a contraction

$$
C: \mathcal{H}_{0} \rightarrow \mathcal{H}_{0}
$$

we observe that if $x \in \mathcal{H}$ and $y \in \mathcal{H}_{0} \ominus(\operatorname{ran} \delta T)^{-}$, then

$$
\|\delta T x+y\|^{2}-\left\|C_{0}(\delta T x+y)\right\|^{2}=\left\langle\triangle_{T} x, x\right\rangle+\|y\|^{2} .
$$

Also note that (5.81) implies the defect identity,

$$
(\delta T)^{*}\left(1-C^{*} C\right) \delta T=\triangle_{T}
$$

In (5.82), $\delta T$ is regarded as an operator into $\mathcal{H}_{0}$.

Now, set

$$
\mathcal{H}_{1}=\left(\operatorname{ran} \triangle_{T}^{\frac{1}{2}}\right)^{-} .
$$

Let $V_{0}: \operatorname{ran} \triangle_{T}^{\frac{1}{2}} \rightarrow \operatorname{ran} \triangle_{T}^{\frac{1}{2}}$ be defined by

$$
V_{0}\left(\triangle_{T}^{\frac{1}{2}} x\right)=\triangle_{T}^{\frac{1}{2}} T x, \quad x \in \mathcal{H} .
$$


Since $T^{*} \triangle_{T} T=\triangle_{T}, V_{0}$ is an isometry and hence extends by continuity to an isometry

$$
V: \mathcal{H}_{1} \rightarrow \mathcal{H}_{1}
$$

Now let $S^{*}$ be a coisometric extension of $C$ acting on a space $\mathcal{K}_{0}$. i.e., $\mathcal{K}_{0}$ is a Hilbert space containing $\mathcal{H}_{0}, S \in \mathcal{L}\left(\mathcal{K}_{0}\right)$ is an isometry such that $\mathcal{H}_{0}$ is invariant for $S^{\text {s* and }}$

$$
C=S^{*} \mid H_{0}
$$

Such a coisometric extension can be obtained from an isometric dilation of $C^{*}[S \%-N-\mathrm{F}]$. Also choose a Hilbert spaco $\mathcal{K}_{1} \supseteq \mathcal{H}_{1}$ and a mitary $U \in \mathcal{L}\left(\mathcal{K}_{1}\right)$ such that $\mathcal{H}_{1}$ is invariant for $U$ and

$$
V=U \mid \mathcal{H}_{1}
$$

Observing that the operators $S$ and $U$ above and the spaces they ast on can be ant ificially enlarged, it is clear that we may assume that

$$
\operatorname{dim}\left(\mathcal{K}_{1} \ni \mathcal{H}_{1}\right)=\operatorname{dim} \mathcal{M}
$$

where

$$
\mathcal{M}=\operatorname{ran}\left(1-S S^{*}\right):\left(\left(1-S S^{*}\right)(\operatorname{ran} \delta T)\right)^{-\cdots}
$$

Now define $E_{0}: \operatorname{ran} \triangle_{T}^{\frac{1}{2}}+\left(\mathcal{K}_{1}-\mathcal{H}_{1}\right) \rightarrow\left(1-S S^{*}\right) \mathcal{K}_{0}$ by choesing a Hilbert space isomorphism $F: \mathcal{K}_{1} \circlearrowleft \mathcal{H}_{1} \rightarrow \mathcal{M}$ and setting

$$
E_{0} \triangle_{T}^{\frac{1}{2}} x=\left(1-S S^{*}\right) \delta T x . \quad x \in \mathcal{H}
$$

and

$$
E_{0} \mid \mathcal{K}_{1}: \mathcal{H}_{1}=F
$$

Observe using (5.83) and (5.84) that

$$
\begin{aligned}
\left\|E_{0} \triangle_{T}^{\frac{1}{2}} x\right\|^{2} & =\left\langle\left(1-S S^{*}\right) \delta T x, \delta T x\right\rangle \\
& =\left\langle\left(1-C^{*} C\right) \delta T x, \delta T x\right\rangle \\
& =\left\langle\triangle_{T} x, x^{\prime}\right\rangle \\
& =\left\|\triangle_{T}^{\frac{1}{2}} x\right\|^{2} .
\end{aligned}
$$

Hence, $E_{0}$ is an isometry and extends by contimuity to a Hilbert space isomorphism

$$
E: \mathcal{K}_{1} \rightarrow\left(1-S S^{*}\right) \mathcal{K}_{0}
$$


Also note that

$$
\left(1-S S^{*}\right) \delta T=E \triangle_{T}^{\frac{1}{2}}
$$

Now note that,

$$
B=\left[\begin{array}{cc}
S & \sigma E \\
0 & U
\end{array}\right]
$$

acting on $\mathcal{K}_{0} \oplus \mathcal{K}_{1}$ is a Brownian unitary of covariance $\sigma$, and that the map $L: \mathcal{H} \rightarrow \mathcal{K}_{0} \oplus \mathcal{K}_{\mathbf{1}}$ defined by

$$
L(x)=\delta x \oplus \frac{1}{\sigma} \triangle_{T}^{\frac{1}{2}} x
$$

is an isometry. Thus, the proof of Theorem 5.80 will be complete if it is shown that

$$
L T=B L
$$

To see (5.86) holds note that the equation has the two components,

$$
\delta T x=S \delta x+E \triangle_{T}^{\frac{1}{2}} x,
$$

and

$$
\frac{1}{\sigma} \triangle_{T}^{\frac{1}{2}} T x=U \frac{1}{\sigma} \triangle_{T}^{\frac{1}{2}} x
$$

for all $x \in \mathcal{H}$. To prove (5.87) note using (5.85), (5.83), and the definition of $C$, that

$$
\begin{aligned}
\delta T x & =S S^{*} \delta T x+\left(1-S S^{*}\right) \delta T x \\
& =S S^{*} \delta T x+E \triangle_{T}^{\frac{1}{2}} x \\
& =S C \delta T x+E \triangle_{T}^{\frac{1}{2}} x \\
& =S \delta x+E \triangle_{T}^{\frac{1}{2}} x .
\end{aligned}
$$

The proof of (5.88) follows in a similar fashion using (5.84) and the definition of $V$. This concludes the proof of Theorem 5.80 .

We will close this section with a theorem that demonstrates the optimality of Theorem 5.80 in any model theory for 2 -isometries based on lifting. Let $\mathcal{F}_{\sigma}$ denote the collection of 2 -isometries $T$ with $\operatorname{cov}(T) \leq \sigma$. Suppose that $\mathcal{B} \subseteq \mathcal{F}_{\sigma}$ is any subcollection with the property that

$$
B \in \mathcal{B} \text { and } \mathcal{M} \text { reducing for } B \text { implies that } B \mid \mathcal{M} \in \mathcal{B} \text {. }
$$

We claim that if $\mathcal{B}$ is a collection of operators providing a model for the general 2 isometry as in Theorem 5.80 (i.e., every element of $\mathcal{F}_{\sigma}$ has an extension to a $B \in \mathcal{B}$ ), then necessarily $\mathcal{B}$ contains the Brownian unitaries of covariance $\sigma$. The heart of the matter rests in the following lemma. 
Lemma 5.90. If $T \in \mathcal{L}(\mathcal{H})$ is a 2-isometry of covariance $\sigma$ and $\mathcal{M} \subseteq \mathcal{H}$ is an invariant subspace for $T$ such that $T \backslash \mathcal{M}$ is a Brownian unitary of covariance $\sigma$, then $\mathcal{M}$ reduces $T$.

Proof. Let $B=T \mid \mathcal{M}$. $T$ has the block representation,

$$
T=\left[\begin{array}{ccc}
V & \sigma E & X \\
0 & U & Y \\
0 & 0 & Z
\end{array}\right]
$$

with respect to the decomposition $\mathcal{H}=\operatorname{ker} \triangle_{B} \oplus \operatorname{ran} \triangle_{B} \oplus \mathcal{M}^{\perp}$ for some operators $X$ : $\mathcal{M}^{\perp} \rightarrow \operatorname{ker} \triangle_{B}, Y: \mathcal{M}^{\perp} \rightarrow \operatorname{ran} \triangle_{B}$ and $Z \in \mathcal{L}\left(\mathcal{M}^{\perp}\right)$. Furthermore since $B$ is a Brownian unitary of covariance $\sigma$,

$$
V \text { is an isometry, }
$$

and

$$
U \text { is unitary . }
$$

To prove the lemma it suffices to show that $X=0$ and $Y=0$.

Now, computing $\triangle_{T}$ gives

$$
\triangle_{T}=\left[\begin{array}{ccc}
0 & 0 & V^{*} X \\
0 & \sigma^{2} & \sigma E^{*} X+U^{*} Y \\
X^{*} V & \sigma X^{*} E+Y^{*} U & X^{*} X+Y^{*} Y+Z^{*} Z-I
\end{array}\right] .
$$

In particular, since $\triangle_{T} \geq 0$,

$$
X^{*} V=0 \text {. }
$$

Also, since $B$ is a Brownian unitary of covariance $\sigma$,

$$
\operatorname{ran} \triangle_{B}=\operatorname{ker}\left(\sigma^{2}-\triangle_{B}\right)
$$

and since $\sigma^{2}-\triangle_{T} \geq 0$ and $B=T \mid \mathcal{M}$,

$$
\operatorname{ker}\left(\sigma^{2}-\Delta_{B}\right) \subseteq \operatorname{ker}\left(\sigma^{2}-\Delta_{T}\right)
$$


Hence, one deduces from (5.96) that

$$
\sigma X^{*} E+Y^{*} U=0
$$

Now using (5.96), (5.97) and (5.98) and the fact $T^{*} \triangle_{T} T-\triangle_{T}=0$ gives

$$
\left[\begin{array}{ccc}
0 & 0 & 0 \\
0 & 0 & \sigma^{2} U^{*} Y \\
0 & \sigma^{2} Y^{*} U & *
\end{array}\right]=0 .
$$

Hence (5.95) implies that $Y=0$.

To see that $X=0$ note that since $Y=0,(5.98)$ implies that $X^{*} E=0$. Hence by (5.94) and (5.97), $X^{*}=0$. This concludes the proof of Lemma 5.90.

Lemma 5.90 does not generalize to Brownian isometries. Indeed, by Lemma 5.78

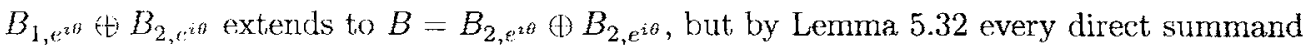
of $B$ is a Brownian unitary.

We now are able to prove our promised result that the Brownian unitaries give an optimal model theory for 2-isometries.

Theorem 5.99. Fix $\sigma>0$. If $\mathcal{B}$ is a subcollection of $\mathcal{F}_{\sigma}$ satisfying property (5.89) and with the property that every clement of $\mathcal{F}_{\sigma}$ has an extension to an element of $\mathcal{B}$, then $\mathcal{B}$ contains the Brownian unitaries of covariance $\sigma$.

ProOf. Suppose $\mathcal{B} \subseteq \mathcal{F}_{\sigma}$ has the properties of the theorem and fix a Brownian unitary $B$ of covariance $\sigma$. Evidently, there exists $T \in \mathcal{B}$ and an invariant subspace $\mathcal{M}$ for $T$ such that $B=T \mid \mathcal{M}$. By Lemma $5.90, \mathcal{M}$ is reducing for $T$. Hence by $(5.89), B \in \mathcal{B}$. This concludes the proof of Theorem 5.99 .

\section{\$6. A Disconjugacy Theorem for Matricial Toeplitz Op- erators}

In this section we shall generalize the results of $[\mathbf{A g 4}]$ to the matricial case. If $L$ is a DDO and the coefficients of $L$ are functions, then the DTO $A=P L \mid \mathcal{D}_{a}$ has an extension to a densely defined self-adjoint operator on $H^{2}$ and this extension is a Toeplitz operator in the sense of Boutet de Monvel and Guillemin [M-G]. In [M-G], working on a general compact, strictly pseudoconvex domain with smooth boundary, the authors (stablish discreteness of spectrum, Fredholm properties and an analysis of the asymptotic behavior of the spectrum for the class of elliptic Toeplitz operators. There is, however, another qualitative phenomenon present in the classical theory of elliptic $O D E$, namely disconjugacy. In [Ag4] it was shown that the classical disconjugacy theorem for 2nd order 
$O D E$ (as well as the related Sturm-Liouville phenomena) generalize to the Toeplitz case where $L$ has the form,

$$
L=D+w
$$

with $w \in L_{\mathbb{R}}^{\infty}(\partial \mathbb{D})$. This result, which implies that certain cyclic 2-isometries possess a cyclic vector which is an eigenfunction for $L$, was then used in [Ag4] to establish Lemma 6.7 below in the special case where $T$ is cyclic and has a "Wiener-Hopf form" as defined in Section 4. In this section we shall generalize the results just described from the scalar to the matrix case. In particular, we establish a disconjugacy theorem for matrix DTO and then use this theorem to show how to concretely calculate a Brownian isometric extension for the 2-isometry $M_{A}$ in terms of the spectral information of $A$.

Recall from Section 4 that if $T \in \mathcal{L}(\mathcal{H})$ is an $n$-cyclic 2 -isometry, then we say that $T$ possesses a Wiener-Hopf form if there exists a cyclic $n$-tuple $\gamma=\left(\gamma_{1}, \ldots, \gamma_{n}\right)$ for $T$ such that the matricial DDO $(T, \gamma)^{\wedge}$ has the form,

$$
(T, \gamma)^{\hat{n}}=\frac{d \theta}{2 \pi} D+\beta_{0}
$$

i.e., $\beta_{1}=\frac{d \theta}{2 \pi}$. Furthermore recall from Theorem 4.18 that any regular 2-isometry possesses a Wiener-Hopf form.

Now, if $L$ is as in (6.1) and if we set $A=P L \mid \mathcal{D}_{a}^{n, 1}$, then $A$ is completely determined by $\beta_{0}$, the intercept of $L$. Furthermore, if $\beta_{0} \in\left(L^{\infty}\right)^{n, n}$, then we may regard $A$ as an unbounded operator on $\left(H^{2}\right)^{n, 1}$. Specifically, if $w \in\left(L^{\infty}\right)^{n, n}$, recall that the Toeplitz operator $T_{w}:\left(H^{2}\right)^{n, 1} \rightarrow\left(H^{2}\right)^{n, 1}$ is defined by

$$
T_{w} f=P(w f)
$$

where $P$ denotes the orthogonal projection of $\left(L^{2}\right)^{n, 1}$ onto $\left(H^{2}\right)^{n, 1}$. It is easy to verify that if $A_{w}$ is defined on $\left(H^{2}\right)^{n, 1}$ by

$$
\begin{aligned}
\operatorname{dom}\left(A_{w}\right) & =\left\{f \in\left(H^{2}\right)^{n, 1}: f^{\prime} \in\left(H^{2}\right)^{n, 1}\right\} \\
A_{w} f & =D f+T_{w} f, f \in \operatorname{dom}\left(A_{w}\right),
\end{aligned}
$$

then $A_{w}$ is an unbounded self adjoint operator. Noting the formula,

$$
\left\langle A_{w} \varphi, \psi\right\rangle=\left(\frac{d \theta}{2 \pi} D+w \frac{d \theta}{2 \pi}\right)(\varphi)\left(\psi^{*}\right), \varphi, \psi \in \mathcal{D}_{a}^{n, 1}
$$

it is clear that the study of $n$-cyclic 2 -isometries that possess a Wiener-Hopf form with bounded intercept corresponds to the study of the operators multiplication by $z$ on the form domain of the operators $A_{w}$ defined in (6.2). 
Accordingly, if $w \in\left(L^{\infty}\right)^{n, n}$ and $A_{w}$ is defined as in (6.2), let us agree to say $A_{w}$ is positive $\left(A_{w} \geq 0\right)$ if

$$
\left\langle A_{w} f, f\right\rangle \geq 0 \text { for all } f \in \operatorname{dom}\left(A_{w}\right) .
$$

Let us agree to say that $A_{w}$ is an analytic Dirichlet operator if there exists a positive constant $\varepsilon$ such that

$$
A_{w}-\varepsilon \geq 0
$$

If $A_{w}$ is positive, define $H_{w}^{2}$, the form domain of $A_{w}$, to be the completion of dom $\left(A_{w}\right)$ with respect to the bilinear form,

$$
[f, g]_{w}=\left\langle A_{w} f, g\right\rangle
$$

Evidently, using (6.3) and Lemma 3.28, $A_{w}$ is an analytic Dirichlet operator if and only if the operator $M_{w}$, defined densely on $H_{w}^{2}$ by

$$
\left(M_{w} f\right)(z)=z f(z), \quad f \in \operatorname{dom}\left(A_{w}\right)
$$

is a well-defined bounded operator. Also, in this event, $H_{w}^{2} \subseteq\left(H^{2}\right)^{n, 1}$ (here, $H^{2}$ denotes the classical Hardy space). It should be clear also that if $A_{w}$ is an analytic Dirichlet operator, then $M_{w}$ is a 2-isometry. If we set $\triangle_{w}=\triangle_{M_{w}}$, then Proposition 3.41 implies that

$$
\left[\triangle_{w} f, g\right]_{w}=\langle f, g\rangle \quad f, g \in H_{w}^{2}
$$

Formula (6.5) has a very interesting consequence that allows one to see why spectral information about $A_{w}$ might have interesting model theoretic consequences for the 2-isometry $M_{w}$. A priori, the spectrum of $A_{w}$ consists entirely of eigenvalues of finite multiplicity clustering at $+\infty$. Thus (6.4) implies that $A_{w}$ is compactly invertible. Formula (6.5) is the assertion that

$$
A_{w}^{-1}=\triangle_{w}
$$

i.e., $A_{w} \triangle_{w} h=h$ for all $h \in H_{w}^{2}$ and $\triangle_{w} A_{w} h=h$ for all $h \in \operatorname{dom}\left(A_{w}\right)$.

We begin with a generalization of Lemma 1.2 from [Ag4].

Lemma 6.7. Let $w \in\left(L^{\infty}\right)^{n, n}$ with $w\left(e^{i \theta}\right)=w\left(e^{i \theta}\right)^{*}$ for a.e $\theta$. If $A_{w} \geq 0$ and $f_{1}, \ldots, f_{k} \in$ $\operatorname{dom}\left(A_{w}\right)$ with $A_{w} f_{r}=0$ for each $r=1, \ldots k$, then the following are equivalent.

(i) $f_{1}, \ldots, f_{k}$ are linearly independent .

(ii) For each $\alpha \in \mathbb{D}, f_{1}(\alpha), \ldots, f_{k}(\alpha)$ are linearly independent. 
(iii) There exists $\alpha \in \mathbb{D}$ such that $f_{1}(\alpha), \ldots, f_{k}(\alpha)$ are linearly independent.

Proof. Observe that (ii) $\Rightarrow$ (iii) and (iii) $\Rightarrow$ (i) are tautologies. To see that (i) $\Rightarrow$ (ii) assume that $f_{1}, \ldots, f_{k}$ are linearly independent. Let $\alpha \in \mathbb{D}$ and fix $c_{1}, \ldots, c_{k} \in \mathbb{C}$ with

$$
\sum_{r=1}^{k} c_{r} f_{r}(\alpha)=0
$$

The proof of Lemma 6.7 will be complete once it is shown that $c_{r}=0$ for each $r$. Set

$$
f(z)=\sum_{r=1}^{k} c_{r} f_{r}(z), \quad z \in \mathbb{D} .
$$

Evidently (6.8) implies that if $B(z)$ is defined by

$$
B(z)=\frac{z-\alpha}{1-\bar{\alpha} z}, \quad z \in \mathbb{D}
$$

then there exists $g \in \operatorname{dom}\left(A_{w}\right)$ such that $f=B g$. Computing as in the proof of Lemma 1.7 in $[\mathbf{A g} 4]$ we find that $g=0$. Thus, $f=0$, and hence, since $f_{1}, \ldots, f_{k}$ are linearly independent, $c_{r}=0$ for each $r$ and the proof of Lemma 6.7 is complete.

Lemma 6.7 has interesting applications for the spectral theory of the covariance operator $\triangle_{T}$ of an $n$-cyclic 2 -isometry $T$. First assume that $T$ is an $n$-cyclic 2 -isometry with cyclic $n$-tuple $\gamma$ and with $(T, \gamma)^{\wedge}$ in Wiener-Hopf form with bounded intercept $w$. Thus, $T$ is unitarily equivalent to $M_{w}$ acting on $H_{w}^{2}$. For $\alpha \in \mathbb{D}$ and $x \in \mathbb{C}^{n}$ choose $k_{\alpha, x} \in H_{w}^{2}$ with the property that

$$
\left[f, k_{\alpha, x}\right]_{w}=\langle f(\alpha), x\rangle_{\mathbb{C}^{n}}, f \in H_{w}^{2} .
$$

There are many ways to deduce the existence and uniqueness of such $k_{\alpha, x}$, but perhaps the most definitive analysis is provided by (6.5) which implies that

$$
k_{\alpha, x}=\Delta_{w} s_{\alpha, x}
$$

where $s_{\alpha, x}$ is the Szegö kernel for $\left(H^{2}\right)^{n, 1}$ (i.e., $s_{\alpha, x}=\frac{x}{1-\bar{\alpha} e^{i \theta}}$ has the property $\left\langle f, s_{\alpha, x}\right\rangle=$ $\langle f(\alpha), x\rangle)_{n}$ for $\left.f \in\left(H^{2}\right)^{n, 1}\right)$.

Now, first observe that (6.6) implies that

$$
\operatorname{ker}\left(A_{w}-\rho_{1}\right)=\operatorname{ker}\left(\left\|\triangle_{w}\right\|-\triangle_{w}\right)
$$

where $\rho_{1}$ denotes the first eigenvalue of $A_{w}$. Sccondly, note that if $\alpha \in \mathbb{D}$, then

$$
f \in \operatorname{ran}\left(M_{w}-\alpha\right) \text { if and only if } f(\alpha)=0
$$


Now assume that $f_{1}, \ldots, f_{k} \in \operatorname{ker}\left(\left\|\triangle_{w}\right\|-\triangle_{w}\right)$ are chosen to be linearly independent. By (6.12) and Lemma 6.7 applied to $A_{w-\rho_{1}}$, no nontrivial linear combination of $f_{1}, \ldots, f_{k}$ vanishes at $\alpha$. Hence, by (6.13), no nontrivial linear combination of $f_{1}, \ldots, f_{k}$ meets $\operatorname{ran}\left(M_{w}-\alpha\right)$. Summarizing, we have shown that

$$
\operatorname{ker}\left(\left\|\triangle_{w}\right\|-\triangle_{w}\right) \cap \operatorname{ran}\left(M_{w}-\alpha\right)=\{0\}
$$

The assertion (6.14) is an intrinsic statement about the covariance operator of an $n$-cyclic 2 -isometry in Wiener-Hopf form with bounded intercept. In fact (6.14) is true for a general nonisometric 2-isometry.

Proposition 6.15. If $T$ is a nonisometric 2-isometry and $\alpha \in \mathbb{D}$, then

$$
\operatorname{ker}\left(\left\|\triangle_{T}\right\|-\triangle_{T}\right) \cap \operatorname{ran}(T-\alpha)=\{0\}
$$

Proof. We leave as an exercise in algebra the fact that Proposition 6.15 holds when $T$ is a nonunitary Brownian unitary. If $T$ is a nonisometric 2-isometry with $\sigma^{2}=\left\|\Delta_{T}\right\|>0$, choose a Brownian unitary extension $B$ for $T$ of covariance $\sigma$ (Theorem 5.80). Evidently,

$$
\operatorname{ker}\left(\sigma^{2}-\triangle_{T}\right) \subseteq \operatorname{ker}\left(\sigma^{2}-\triangle_{B}\right)
$$

and

$$
\operatorname{ran}(T-\alpha) \subseteq \operatorname{ran}(B-\alpha)
$$

Since

$$
\operatorname{ker}\left(\sigma^{2}-\triangle_{B}\right) \cap \operatorname{ran}(B-\alpha)=\{0\},
$$

the conclusion of Proposition 6.15 follows.

We remark before continuing that by the manner in which we prove Proposition 6.15 we have shown that the lifting theorem (Theorem 5.80) implies the disconjugacy theorem (Lemma 6.7). Conversely, in Theorem 6.20 we shall see how the disconjugacy theorem implies the lifting theorem.

Our next result bears the same relation to Lemma 1.2 in [Ag4] that the lemma just proved bears to Lemma 1.7 in [Ag4]. The differences between Lemma 6.16 below and Lemma 6.7 are that now $\alpha \in \partial \mathbb{D}$ (rather than $\alpha \in \mathbb{D}$ ) and the DTO $A_{w}$ is not assumed to be positive definite.

Lemma 6.16. Let $w \in\left(L^{\infty}\right)^{n, n}$ with $w\left(e^{i \theta}\right)=w\left(e^{i \theta}\right)^{*}$ for a.e. $e^{i \theta}$. If $f_{1}, \ldots, f_{k} \in$ $\operatorname{dom}\left(A_{w}\right)$ with $A_{w} f_{r}=0$ for each $r=1, \ldots, k$, then the following three statements are equivalent.

(i) $f_{1}, \ldots, f_{k}$ are linearly independent. 
(ii) For each $\alpha \in \partial D$, $f_{1}(\alpha), \ldots, f_{k}(\alpha)$ are linearly independent.

(iii) There exists $\alpha \in \partial \mathbb{D}$ such that $f_{1}(\alpha), \ldots, f_{k}(\alpha)$ are linearly independent.

Proof. As in the proof of Lemma 6.7, it suffices to prove that (i) $\Rightarrow$ (ii). Accordingly, assume that $f_{1}, \ldots f_{k}$ are linearly independent. Let $\alpha \in \partial \mathbb{D}$ and fix $c_{1}, \ldots, c_{k} \in \mathbb{C}$ such that (6.8) holds. If $f$ is defined as in (6.9), then, as in the proof of Lemma 1.2 from [Ag4], there exists $g \in\left(H^{2}\right)^{n, 1}$ such that $f=(z-\alpha) g$. Also as in the proof of Lemma 1.7, the equation

$$
0=\left\langle A_{w}(z-\alpha) g,(z+\alpha) g\right\rangle
$$

implies that $g=0$. Since $g=0$, so also $f=0$ and since $f_{1}, \ldots, f_{k}$ are linearly independent, we deduce that $c_{r}=0$ for each $r$. This concludes the proof of Lemma 6.16.

Lemma 6.7 and 6.16 have many interesting implications for both the theory of first order Toeplitz operators as well as the theory of 2-isometries. We content ourselves with the following proposition.

Proposition 6.17. If $w \in\left(L^{\infty}\right)^{n, n}, w\left(e^{i \theta}\right)=w\left(e^{i \theta}\right)^{*}$ for almost every $e^{i \theta} \in \partial \mathbb{D}$, and $A_{w} \geq 0$, then dim ker $A_{w} \leq n$. If $f_{1}, \ldots, f_{k} \in$ ker $A_{w}$ are linearly independent, then $f_{1}(\alpha), \ldots, f_{k}(\alpha)$ are linearly independent for each $\alpha \in \mathbb{D}^{-}$.

Now, Proposition 6.17 will turn out to have a remarkable interpretation (6.24 below) for the case when $T$ is a 2 -isometry of the form $T=M_{w}$. We begin with a characterization of $H_{w}^{2}$ when $u \in\left(L^{\infty}\right)^{n, n}$. Note that with our current notation $H_{1}^{2}$ is the direct sum of $n$ copies of the Dirichlet space.

Proposition 6.18. If $w \in\left(L^{\infty}\right)^{n, n}$ and $A_{w}$ is an analytic Dirichlet operator, then there cxist constants $c_{1}, c_{2}>0$ such that

$$
c_{1}\|\varphi\|_{A_{1}} \leq\|\varphi\|_{A_{u}} \leq c_{2}\|\varphi\|_{A_{1}}
$$

for all $\varphi \in \mathcal{D}_{a}^{n, 1}$. In particular, $M_{w}$ is similar to a direct sum of $n$ copies of the Dirichlet shift, and if $F=\left(f_{r s}\right)$ is a matrix of analytic functions with $f_{r s}^{\prime} \in H^{2}$ for all $r, s \leq n$, then $F$ is a multiplier of $H_{w}^{2}$.

Proor. The latter assertion of the proposition follows from (6.19) and the fact that if $f^{\prime} \in H^{2}$, then $f$ is a multiplier of the Dirichlet space.

To prove (6.19) first note that if $\varphi \in \mathcal{D}_{a}^{n, 1}$, then

$$
\begin{aligned}
\|\varphi\|_{A_{w}}^{2}= & \langle D \varphi, \varphi\rangle+\langle u \varphi, \varphi\rangle \\
& \leq \max \left\{1,\|w\|_{\infty}\right\}\|\varphi\|_{A_{1}}^{2}
\end{aligned}
$$


Secondly, note that since

$$
\begin{aligned}
\langle(1-w) \varphi, \varphi\rangle & \leq\|1-w\|_{\infty}\langle\varphi, \varphi\rangle \\
= & \|1-w\|_{\infty}\left[\triangle_{w} \varphi, \varphi\right]_{w} \\
& \leq\left\|\triangle_{w}\right\|\|1-w\|_{\infty}\|\varphi\|_{w}^{2}
\end{aligned}
$$

we obtain that

$$
\begin{aligned}
\|\varphi\|_{A_{1}}^{2}= & \langle D \varphi, \varphi\rangle+\langle\varphi, \varphi\rangle \\
= & \|\varphi\|_{A_{w}}^{2}+\langle(1-w) \varphi, \varphi\rangle \\
& \leq\left(1+\left\|\triangle_{w}\right\|\|1-w\|_{\infty}\right)\|\varphi\|_{A_{w}}^{2} .
\end{aligned}
$$

Thus (6.19) holds with $c_{2}=\max \left\{1,\|w\|_{\infty}\right\}^{\frac{1}{2}}$ and $c_{1}=\left(1+\left\|\triangle_{w}\right\|\|1-w\|_{\infty}\right)^{-\frac{1}{2}}$ and the proof of Proposition 6.18 is complete.

The remainder of this section will be devoted to proving the following theorem.

Theorem 6.20. Let $w \in\left(L^{\infty}\right)^{n, n}$ be such that $w\left(e^{i \theta}\right)=w\left(e^{i \theta}\right)^{*}$ for a.e. $e^{i \theta}$ and such that $A_{w}$ is an analytic Dirichlet operator with $\left\|\triangle_{w}\right\|=\sigma^{2}$ (equivalently, the first eigenvalue of $A_{w}$ is $\left.\sigma^{-2}\right)$. There exists $v \in\left(L^{\infty}\right)^{n, n}$ such that

$$
v\left(e^{i \theta}\right) \geq \sigma^{-2} \quad \text { for } \text { a.e. } \quad e^{i \theta} \in \partial \mathrm{DD}
$$

$$
A_{w-v} \geq 0 \text { and }
$$

If $v \in\left(L^{\infty}\right)^{n . n}$ is any matrix function satisfying (6.21) - (6.23), then

$$
\text { ker } A_{w-v} \text { is cyclic for } M_{w} \text {. }
$$

Furthermore, if $v \in\left(L^{\infty}\right)^{n, n}$ is any matrix function satisfying (6.21)-(6.24), if $f_{1}, \ldots, f_{n}$ is any basis for $\operatorname{ker}\left(A_{w-v}\right)$ and if $B$ is defined by

$$
B=\int_{\oplus} B_{v\left(e^{i \theta}\right)^{-\frac{1}{2}}, e^{i \theta}} \frac{d \theta}{2 \pi}
$$

then $B$ has covariance $\sigma$ and the slope and intercept sequences of $\left(M_{w} ; f_{1}, \ldots, f_{n}\right)$ and

$$
\left(B ; \int_{\oplus}\left[v\left(e^{i \theta}\right)^{\frac{1}{2}} f_{1}\left(e^{i \theta}\right)\right] \frac{d \theta}{2 \pi}, \ldots, \int_{\oplus}\left[\begin{array}{c}
0 \\
v\left(e^{i \theta}\right)^{\frac{1}{2}} f_{n}\left(e^{i \theta}\right)
\end{array}\right] \frac{d \theta}{2 \pi}\right)
$$


are the same. In particular, $M_{w}$ has an extension to a Brownian unitary of covariance $\sigma$.

The remainder of this section will be devoted to the proof of Theorem 6.20 . We first construct an element $v \in\left(L^{\infty}\right)^{n, n}$ with the properties (6.21), (6.22) and (6.23). Once this is accomplished we invoke Proposition 6.17 to deduce that if $n$ vectors $f_{1}, \ldots, f_{n} \in$ $\operatorname{ker}\left(A_{w-v}\right)$ are chosen linearly independent, then $\left(f_{1}, \ldots, f_{n}\right)$ is a cyclic n-tuple for $M_{w}$. Finally, the proof of 6.20 will be completed by a direct calculation using the formulas

$$
A_{w} f_{r}=P\left(v f_{r}\right) \quad r=1, \ldots, n \text {. }
$$

To construct $v \in\left(L^{\infty}\right)^{n, n}$ with the desired properties we shall proceed by induction using the following simple perturbation lemma.

Lemma 6.26. Suppose $u \in\left(L^{\infty}\right)^{n, n}, A_{u} \geq 0$ and $\operatorname{dim}$ ker $A_{u}=k$. If $0 \leq k<n$, then there exists $v \in\left(L^{\infty}\right)^{n, n}$ such that

$$
\begin{gathered}
v \geq 0, \\
A_{u-v} \geq 0,
\end{gathered}
$$

and

$$
\operatorname{dim} \text { ker } A_{u-v}>k \text {. }
$$

Proof. Let $f_{1}, \ldots, f_{k}$ span ker $A_{u}$. Let $P\left(e^{i \theta}\right)$ denote the orthogonal projection of $\mathbb{C}^{n}$ onto $\left\{f_{1}\left(e^{i \theta}\right), \ldots, f_{k}\left(e^{i \theta}\right)\right\}^{\perp} \subseteq \mathbb{C}^{n}$. Thus, $P \in\left(L^{\infty}\right)^{n, n}, P\left(e^{i \theta}\right) \geq 0$ for almost every $e^{i \theta} \in \partial \mathbb{D}$ (in fact, $P$ is continuous) and

$$
\operatorname{ran} T_{P} \subseteq \operatorname{ran} A_{u}
$$

By the discreteness of the spectrum of $A_{u}$, it follows that there exists a largest positive constant $t$ such that

$$
t T_{P} \leq A_{u}
$$

The proof of Lemma 6.26 is completed by letting $v=t P$.

The existence of $v \in\left(L^{\infty}\right)^{n, n}$ satisfying (6.21), (6.22) and (6.23) now follows by considering the following set $S$ :

$S=\left\{k \geq 1\right.$ : there exists $v \in\left(L^{\infty}\right)^{n, n}$ such that (6.21) and (6.22) holds and such that $\left.\operatorname{dim} \operatorname{ker} A_{w-v}=k\right\}$.

First note that (6.6) implies that $\sigma^{-2}$ is the first eigenvalue of $A_{w}$. Hence if $v$ is defined by $v\left(e^{i \theta}\right) \equiv \sigma^{-2}$, then (6.21) and (6.22) hold. Thus, $\operatorname{dim}$ ker $A_{w-\sigma^{-2}} \in S$. On the other hand, Lemma 6.26 asserts that if $1 \leq k<n$ and $k \in S$, then there exists $k_{1}>k$ with $k_{1} \in S$. Finally, Proposition 6.17 asserts that if $k \in S$, then $k \leq n$. Hence $n \in S$ and a $v$ with the desired properties exists. 
Now assume that $v \in\left(L^{\infty}\right)^{n, n}$ is any matrix function satisfying (6.21)-(6.23). Choose $n$ vectors $f_{r} \in\left(H^{2}\right)^{n, 1}$ with ker $A_{w-v}=\bigvee\left\{f_{r}: 1 \leq r \leq n\right\}$. We claim that $\left(f_{1}, \ldots, f_{n}\right)$ is a cyclic $n$-tuple for $M_{w}$. First observe that since $f_{r}^{\prime} \in\left(H^{2}\right)^{n, 1}$, Proposition 6.18 implies that $f_{r}$ is in fact an element of $H_{w}^{2}$. Now let $f_{r s}(z)$ denote the $s^{t h}$ component of $f_{r}(z)$ and define $F \in(\operatorname{Hol}(\mathbb{D}))^{n, n}$ by

$$
F(z)=\left(f_{r s}(z)\right) .
$$

Since $f_{r}^{\prime} \in\left(H^{2}\right)^{n, 1}, F \in C\left(\mathbb{D}^{-}\right)^{n, n}$, and Propositions 6.17 and 6.18 imply that $F^{-1}$ is a multiplier of $H_{w}^{2}$. Since $F^{-1} f_{r}=e_{r}$ (where the $j$ th component of $e_{r}$ is 0 if $j \neq r$ and is the constant function 1 if $j=r)$, we deduce that $\left(f_{r}\right)$ is a cyclic $n$-tuple for $M_{w}$.

We now conclude the proof of Theorem 6.20. Let $v \in\left(L^{\infty}\right)^{n, n}$ satisfy (6.21)-(6.23), fix a basis $f_{1}, \ldots, f_{n}$ for ker $A_{w-v}$, and define a Brownian isometry $B$ by the formula,

$$
B=\int_{\oplus} B_{v\left(e^{i \theta}\right)^{-\frac{1}{2}}, e^{i \theta}} \frac{d \theta}{2 \pi} .
$$

If vectors $x_{1}, \ldots, x_{n}$ are defined by

$$
x_{r}=\int_{\oplus}\left[\begin{array}{c}
0 \\
v\left(e^{i \theta}\right)^{\frac{1}{2}} f_{r}\left(e^{i \theta}\right)
\end{array}\right] \frac{d \theta}{2 \pi},
$$

then a straightforward calculation using formula (6.25) gives that

$$
\left\{\begin{array}{l}
\left\langle B^{k} x_{r}, x_{s}\right\rangle=\left\langle M_{w}^{k} f_{r}, f_{s}\right\rangle, \quad \text { and } \\
\left\langle\triangle_{B} B^{k} x_{r}, x_{s}\right\rangle=\left\langle\triangle_{M_{w}} M_{w}^{k} f_{r}, f_{s}\right\rangle .
\end{array}\right.
$$

Now, if $f=\left(f_{r}\right)$ and $x=\left(x_{r}\right)$, then (6.27) implies that $(B, x)^{\wedge}=\left(M_{w}, f\right)^{\wedge}$. Hence since $f$ is a cyclic tuple for $M_{w}$, Theorem 3.49 implies that there exists an isometry $L$ such that

$$
\begin{aligned}
L T & =B L, \quad \text { and } \\
L\left(f_{r}\right) & =x_{r}, \quad \text { for each } r .
\end{aligned}
$$

We claim that $\operatorname{cov}(B)=\sigma$. To see this simply note that (6.21) implies that $\operatorname{cov}(B) \leq \sigma$, and $(6.28)$ implies that $\operatorname{cov}(B) \geq \sigma$.

Finally, we note that (6.28) implies that $T$ has a Brownian isometric extension of covariance $\sigma$. A Brownian unitary extension of covariance $\sigma$ for $T$ is obtained by an application Proposition 5.79.

\section{§7. Another proof of the Lifting theorem}

In this section we shall show how to prove Theorem 5.80 using the disconjugacy results of the previous section. This proof of Theorem 5.80 is considerably more involved than the algebraic proof given in Section 5 and imparts correspondingly more structural information. It was the way the theorem was originally discovered and proven and the proof technique 
has considerably greater power than is present in the proof given in Section 5. This proof technique involves an application of the Arveson extension theorem for completely positive maps $[\mathbf{A r v 1}, \mathbf{A r v 2}]$ to a certain self adjoint subspace $H_{\sigma}$ of a $C^{*}$-algebra $C_{\sigma}$ that is defined in a concrete way using the one parameter family of Brownian shifts $B_{\sigma, e^{i \theta}}$. Specifically, $C_{\sigma}$ is the $C^{*}$-subalgebra of $\mathcal{L}\left(\int\left(H^{2} \oplus \mathbb{C}\right) \frac{d \theta}{2 \pi}\right)$ generated by $c_{\sigma}$ where $c_{\sigma}$ is defined by

$$
c_{\sigma}=\int_{\oplus} B_{\sigma, e^{i \theta}} \frac{d \theta}{2 \pi}
$$

and $H_{\sigma}$ is the self adjoint subspace of $C_{\sigma}$ generated by

$$
\left\{c_{\sigma}^{*^{j}} c_{\sigma}^{i}: i, j \geq 0\right\}
$$

It turns out that an operator $B \in \mathcal{L}(\mathcal{H})$ is a Brownian unitary of covariance $\sigma$ if and only if $B=\pi\left(c_{\sigma}\right)$ for some unital ${ }^{*}$-representation of $C_{\sigma}$ on $\mathcal{H}$ (Lemma 7.9). This fact then allows one to see via work of Stinespring [Sti] and the work of Arveson earlier referred to that an operator $T$ has an extension to a Brownian unitary of covariance $\sigma$ if and only if the map

$$
H_{\sigma} \ni h\left(c_{\sigma}\right) \mapsto h(T)
$$

is completely positive. Thus, modulo $C^{*}$-algebra the spatial question of whether or not every 2-isometry lifts to a Brownian unitary reduces to the analytical question of whether or not the map defined in (7.0) is completely positive whenever $T$ is a 2-isometry of covariance $\sigma$ (Theorem 7.12). This idea of using $C^{*}$-algebra to reduce lifting questions to concrete analytical issues was first used in [Ag1] to show that 3-symmetric operators have extensions to jordan blocks. Other applications of the proof technique have been in the following papers: [Ag2], an application to coanalytic models; [Ag5], an application to sub n-normal operators; [Ag6], an application to hypercontractive and subnormal operators; [Ag7], an application to annular spectral sets; [M1], [M2], applications to 3-isometries and subbrownian operators; $[\mathbf{F}]$, an application to polynomially subnormal tuples; $[\mathbf{C}-\mathbf{P}]$, an application to polynomially hyponormal operators; [St], an application to isosymmetric: operators.

The remainder of the section is devoted to establishing that indeed the map defined in (7.0) is completely positive. A first observation is that it suffices to show completely positivity in the case where $T$ is a finitely cyclic 2-isometry $T$ (Reduction 1 below). It turns out that the complete positivity of (7.0) in this case is stable under the convolution operation on the underlying DDO of $T$ introduced in Section 4 (Reduction 2 below). This latter question is then resolved by a direct calculation using Theorem 6.20 . 
For $m$ and $n$ positive integers, let $\mathbb{C}^{m, n}\{x, y]$ denote the polynomials in $x$ and $y$ with $m \times n$ matrix coefficients. If

$$
h=\sum c_{i j} y^{j} x^{i} \in \mathbb{C}^{m, n}[x, y]
$$

and $a$ is an element of a $C^{*}$-algebra $A$ with unit, then define $h(a) \in A^{m, n}$ (here, $A^{m, n}$ is the space of $m \times n$ matrices with entries in $A$ ) by

$$
(h(a))_{r s}=\sum_{i, j}\left(c_{i j}\right)_{r s} a^{* j} a^{i}, \quad 1 \leq r \leq m, 1 \leq s \leq n
$$

We record for future use that with this notation the following facts hold. Let $\mathcal{H}$ be a complex Hilbert space. Let $T \in \mathcal{L}(\mathcal{H})$ and fix $h \in \mathbb{C}^{n, n}[x, y]$.

(7.1) If $\pi: \mathcal{L}(\mathcal{H}) \rightarrow \mathcal{L}(\mathcal{K})$ is a unital *-representation then $h(\pi(T))=$ $\left(\operatorname{id}_{n} \gg \pi\right)(h(T))$.

(7.2) If $\mathcal{M} \subseteq \mathcal{H}$ is invariant for $T$, then $h(T \mid \mathcal{M})=P_{\mathcal{M}^{(n)}} h(T) \mid \mathcal{M}^{(n)}$.

Furthermore, if for $f \in \mathbb{C}^{m, n}[x, y]$, we define $f^{\sim} \in \mathbb{C}^{n, m}[x, y]$ by setting

$$
f^{\varkappa}(z, w)=f(\bar{w}, \bar{z})^{*}, \quad z, w \in \mathbb{C},
$$

then

$$
h(T)^{*}=\breve{h}(T)
$$

and

$$
\text { If } f \in \mathbb{C}^{n, m}[x] \text { and } \gamma \in \mathcal{H}^{m, 1} \text {, then }\langle h(T) f(T) \gamma, f(T) \gamma\rangle=\left\langle\left(f^{\check{2}} h f\right)(T) \gamma, \gamma\right\rangle \text {. }
$$

In (7.4), $f(T) \gamma$ is defined as in (3.43). Now if $L \in \mathrm{DDO}_{m}^{n, n}$, let $u$ denote the matrix distribution on the torus defined in (3.36), and then define a matrix distribution $u_{L}$ by setting

$$
u_{L}(\varphi)=u\left(\varphi^{\tau}\right), \quad \varphi \in \mathcal{D}^{n, n}
$$

It is casy to see using (3.36) and the definition of $L_{t}$ that $u_{L_{t}}=\left(u_{L}\right)_{t}$. Consequently, one also sees that if $\omega \in \mathcal{D}$, then

$$
u_{\omega * L}=\omega * u_{L} .
$$


Lemma 7.7. If $T \in \mathcal{L}(\mathcal{H})$ is an m-isometry, $\gamma \in \mathcal{H}^{n, 1}$, and $L=(T, \gamma)^{\wedge}$, then

$$
\langle h(T) \gamma, \gamma\rangle=u_{L}\left(h\left(e^{i \theta_{1}}, e^{-i \theta_{2}}\right)\right)
$$

for all $h \in \mathbb{C}^{n, n}[x, y]$.

Proof. If $\varphi, \psi \in \mathbb{C}^{1, n}[x]$, then $\psi^{\nu} \varphi \in \mathbb{C}^{n, n}[x, y]$. Furthermore, polynomials of the form $\psi \varphi \operatorname{span} \mathbb{C}^{n, n}[x, y]$. Hence by linearity it suffices to establish Lemma 7.7 for the case when $h=\tilde{\psi} \varphi$. Let $u$ be the distribution of (3.36). Calculating, we find that

$$
\begin{aligned}
\langle h(T) \gamma, \gamma\rangle & =\langle(\dot{\psi} \varphi)(T) \gamma, \gamma\rangle \\
& =\langle\varphi(T) \gamma, \psi(T) \gamma\rangle \\
& =L\left(\varphi^{\tau}\right)\left(\psi^{\tau *}\right) \\
& =u\left(\varphi^{\tau}\left(e^{i \theta_{1}}\right) \psi^{\tau}\left(e^{i \theta_{2}}\right)^{*}\right) \\
& =u\left(\left(\psi\left(e^{i \theta_{2}}\right)^{*} \varphi\left(e^{i \theta_{1}}\right)\right)^{\tau}\right) \\
& =u_{L}\left(\psi\left(e^{i \theta_{2}}\right)^{*} \varphi\left(e^{i \theta_{1}}\right)\right) \\
& =u_{L}\left(\psi\left(e^{-i \theta_{2}}\right) \varphi\left(e^{i \theta_{1}}\right)\right) \\
& =u_{L}\left(h\left(e^{i \theta_{1}}, e^{-i \theta_{2}}\right)\right),
\end{aligned}
$$

which establishes Lemma 7.7 .

The proof of Theorem 5.80 will be accomplished by a concrete application of the following abstract result from $[\mathbf{A g 2}, \mathbf{A g 3}]$.

Theorem 7.8. Let $C$ be a $C^{*}$-algebra with unit and fix $c \in C$. An operator $T \in \mathcal{L}(\mathcal{H})$ has the form

$$
\pi(c) \mid \mathcal{H}
$$

where $\pi: C \rightarrow \mathcal{L}(\mathcal{K})$ is a unital $*$-representation (i.e., $\pi$ is an algebra homomorphism, $\pi\left(x^{*}\right)=\pi(x)^{*}$ for all $x \in C$, and $\left.\pi(1)=1\right), \mathcal{K} \supseteq \mathcal{H}$, and $\mathcal{H}$ is invariant for $\pi(c)$ if and only if $h(T) \geq 0$ whenever $m \geq 1, h \in \mathbb{C}^{m, m}[x, y]$ and $h(c) \geq 0$.

Theorem 7.8 is applied in our present circumstance as follows. Define $c_{\sigma} \in \mathcal{L}\left(\int_{\oplus} H^{2} \oplus \mathbb{C} \frac{d \theta}{2 \pi}\right)$ by

$$
c_{\sigma}=\int_{\oplus \oplus} B_{\sigma, e^{i \theta}} \frac{d \theta}{2 \pi},
$$

and let $C_{\sigma}$ denote the $C^{*}$-subalgebra of $\mathcal{L}\left(\int_{\oplus} H^{2} \oplus \mathbb{C} \frac{d \theta}{2 \pi}\right)$ generated by $c_{\sigma}$. With the definitions above, the operators $\pi(c)$ in Theorem 7.8 consist precisely of the Brownian unitaries of covariance $\sigma$. 
Lemma 7.9. $B \in \mathcal{L}(\mathcal{H})$ is a Brownian unitary of covariance $\sigma$ if and only if there exists a unital *-representation $\pi: C_{\sigma} \rightarrow \mathcal{L}(\mathcal{H})$ such that $\pi\left(c_{\sigma}\right)=B$.

Proof. First note that Theorem 5.20 implies immediately that $\pi\left(c_{\sigma}\right)$ is a Brownian unitary of covariance $\sigma$ whenever $\pi$ is a unital *-representation. Conversely, if $B \in \mathcal{L}(\mathcal{H})$ is a Brownian unitary of covariance $\sigma$, then by Definition 5.11 , there exists a positive measure $\mu$ supported on $\partial \mathrm{D}$ and a measurable multiplicity function $n_{\theta}$ such that

$$
B \cong \int_{\oplus} B_{\sigma, e^{\theta \theta}}^{\left(n_{\theta}\right)} d \mu(\theta) \oplus U
$$

Now, notice (using the fact that $\theta \mapsto h\left(B_{\sigma, e^{i \theta}}\right)$ is continuous) that $c_{\sigma}$ is constructed so as to have the property,

(7.11) If $m \geq 1$ and $h \in \mathbb{C}^{m, m}[x, y]$, then $h\left(c_{\sigma}\right) \geq 0$ if and only if $h\left(B_{\sigma, \epsilon^{i \theta}}\right) \geq 0$ for all $\theta \in[0,2 \pi)$.

Combining (7.10) and (7.11) we thus are able to deduce that for each $m \geq 1$, if $h \in \mathbb{C}^{m, m}[x, y]$ and $h\left(c_{\sigma}\right) \geq 0$, then $h(B) \geq 0$. Consequently, by Theorem 7.8 , there exists a Hilbert space $\mathcal{K} \supseteq \mathcal{H}$ and a unital $*$-representation $\pi: C_{\sigma} \rightarrow \mathcal{L}(\mathcal{K})$ such that $\mathcal{H}$ is invariant for $\pi\left(c_{\sigma}\right)$ and $B=\pi\left(c_{\sigma}\right) \mid \mathcal{H}$. Since $B$ and $\pi\left(c_{\sigma}\right)$ are Brownian unitaries of covariance $\sigma$, Theorem 5.90 implies that $\mathcal{H}$ is reducing for $\pi\left(c_{\sigma}\right)$. Since $c_{\sigma}$ generates $C_{\sigma}$, $\mathcal{H}$ is in fact reducing for $\pi(x)$ for every $x \in C_{\sigma}$. Hence the formula

$$
\pi_{0}(x)=\pi(x) \mid \mathcal{H}, \quad x \in C_{\sigma}
$$

defines a unital $*$-representation $\pi_{0}: C_{\sigma} \rightarrow \mathcal{L}(\mathcal{H})$. Since $\pi_{0}\left(c_{\sigma}\right)=n\left(c_{\sigma}\right) \mid \mathcal{H}=B$, this concludes the proof of Lemma 7.9 .

Accordingly, we obtain the following corollary of Theorem 7.8 .

Theorem 7.12. An operator $T$ has an extension to a Brownian unitary of covariance $\sigma$ if and only if

$$
h\left(c_{\sigma}\right) \geq 0 \Rightarrow h(T) \geq 0 \text { for all } m \geq 1 \text { and all } h \in \mathbb{C}^{m, m}[x, y]
$$

We now prove Theorem 5.80. Fix a 2-isometry $T$ of covariance $\sigma$. By Theorem 7.12 we need to show that (7.13) holds for $T$.

The proof that condition (7.13) holds for $T$ will consist of a sequence of three reductions culminating in needing only to establish (7.13) in the case when $T$ is a finitely cyclic 2-isometry possessing a Wiener-Hopf form with bounded intercept. For this special case (7.13) is established by appeal to Theorem 6.20. A simple fact which we shall require is the following. 
Lemma 7.14. If $\sigma_{0} \leq \sigma_{1}, m \geq 1, h \in \mathbb{C}^{m, m}[x, y]$, and $h\left(c_{\sigma_{1}}\right) \geq 0$, then $h\left(c_{\sigma_{0}}\right) \geq 0$.

Proof. Let $\mathcal{H}=\int_{\oplus} H^{2} \oplus \mathbb{C} \frac{d \theta}{2 \pi}$. By Proposition 5.79 there exists a Brownian unitary $B$ of covariance $\sigma_{1}$ such that

$$
c_{\sigma_{0}}=B \mid \mathcal{H}
$$

Since $h\left(c_{\sigma_{1}}\right) \geq 0$, Lemma 7.9 implies $h(B) \geq 0$. Hence by $(7.2), h\left(c_{\sigma_{0}}\right) \geq 0$.

For our first reduction we claim that it it enough to show that (7.13) holds when $T$ is finitely cyclic.

Reduction 1. If (7.13) holds for all finitely cyclic 2-isometries $T$ of covariance $\sigma$, then (7.13) holds for all 2-isometries $T$ of covariance $\sigma$.

Proof. Note that if $T \in \mathcal{L}(\mathcal{H}), m \geq 1$ and $h \in \mathbb{C}^{m, m}[x, y]$, then

$$
h(T) \geq 0 \quad \text { if and only if }\langle h(T) \gamma, \gamma\rangle \geq 0 \text { for all } \gamma \in \mathcal{H}^{m, 1}
$$

Since by (7.2)

$$
\left.\left.\langle h(T) \gamma, \gamma\rangle=\langle h(T)| \mathcal{N}_{\gamma}\right) \gamma, \gamma\right\rangle
$$

it follows that

$$
h(T) \geq 0 \text { if and only if } h\left(T \mid \mathcal{N}_{\gamma}\right) \geq 0 \text { for all } \gamma \in \mathcal{H}^{m, m}
$$

Now, let $T \in \mathcal{L}(\mathcal{H})$ be a 2-isometry of covariance $\sigma$. If $m \geq 1$, and $\gamma_{1}, \ldots, \gamma_{m} \in \mathcal{H}$, then $T \mid \mathcal{N}_{\gamma}$ is an $m$-cyclic 2-isometry of covariance $\sigma_{0} \leq \sigma$ so that by Lemma 7.14 and by assumption, (7.13) holds for $T \mid \mathcal{N}_{\gamma}$. Hence, if $m \geq 1, h \in \mathbb{C}^{m, m}[x, y]$, and $h\left(c_{\sigma}\right) \geq 0$, $h\left(T \mid \mathcal{N}_{\gamma}\right) \geq 0$ for all $\gamma \in \mathcal{H}^{m, 1}$. Thus, by (7.15), $h(T) \geq 0$. This shows that (7.13) holds for $T$ and completes the proof of Reduction 1.

Our next reduction uses an approximate identity to approximate the general finitely cyclic 2 -isometry by regular 2 -isometries.

Reduction 2. If (7.13) holds for all $n$-cyclic 2 -isometries $T$ of covariance $\sigma$ such that $(T, \gamma)$ is regular for some cyclic $n$-tuple $\gamma$, then (7.13) holds for all $n$-cyclic 2 -isometries $T$ of covariance $\sigma$.

ProOf. Fix $T \in \mathcal{L}(\mathcal{H})$ an $n$-cyclic 2 -isometry of covariance $\sigma$ and fix $\gamma \in \mathcal{H}^{n .1}$, a cyclic $n$-tuple for $T$.

Let $L=(T, \gamma)^{\hat{n}}$, set $A=P L \mid \mathcal{D}^{n, 1}$ and fix an approximate identity $\left\{\omega_{j}\right\} \subset \mathcal{D} \quad\left(\omega_{j}>\right.$ 0). Using (3.25) it is easy to check that if $A_{j, \epsilon}$ is defined by

$$
A_{j, \epsilon}=P\left(L+\frac{1}{j}(\epsilon D+1)\right) \mid \mathcal{D}_{a}^{n, 1}
$$


then there exists an $\epsilon_{0}>0$ such that $A_{j, \epsilon_{0}}$ is an analytic Dirichlet operator and $\operatorname{cov}\left(A_{j, \epsilon_{0}}\right) \leq \operatorname{cov}(A)=\sigma$ for all $j$.

Now, if $A_{j}$ is defined by

$$
A_{j}=\omega_{j} * A_{j, \epsilon_{0}}
$$

then Proposition 4.11 implies that $A_{j}$ is an analytic Dirichlet operator and $\operatorname{cov}\left(A_{j}\right) \leq \sigma$. Since

$$
\frac{d}{d D} A_{j, \epsilon_{0}}=\frac{d}{d D} A+\frac{\epsilon_{0}}{j} \frac{d \theta}{2 \pi}
$$

is weakly definite, Proposition 4.15 implies that $A_{j}$ is regular. Consequently, by the hypothesis of Reduction 2 and Lemma 7.14, (7.13) holds for $M_{A_{j}}$.

We now show that (7.13) holds for $T$. Accordingly, fix $m \geq 1, h \in \mathbb{C}^{m, m}[x, y]$ and assume that $h\left(c_{\sigma}\right) \geq 0$. We need to show that $h(T) \geq 0$. Since the vectors of the form $F(T) \gamma$ are dense in $\mathcal{H}^{m, 1}$ it suffices to show that

$$
\langle h(T) F(T) \gamma, F(T) \gamma\rangle \geq 0
$$

whenever $F \in \mathcal{D}_{a}^{m, n}$. But

$$
\begin{aligned}
& \langle h(T) F(T) \gamma, F(T) \gamma\rangle=\langle(F \check{h} F)(T) \gamma, \gamma\rangle \\
& =u_{L}\left(F \breve{h} F\left(e^{i \theta_{1}}, e^{-i \theta_{2}}\right)\right) \\
& =\lim _{j \rightarrow \infty}\left(\omega_{j} * u_{L}\right)\left(F^{\top} h F\left(e^{i \theta_{1}}, e^{-i \theta_{2}}\right)\right) \\
& =\lim _{j \rightarrow \infty} u_{\omega_{j} * L}\left(F^{\swarrow} h F\left(e^{i \theta_{1}}, e^{-i \theta_{2}}\right)\right) \\
& =\lim _{j \rightarrow \infty} u_{L_{j}}\left(F^{\wedge} h F\left(e^{i \theta_{1}}, e^{-i \theta_{2}}\right)\right) \quad\left(A_{j}=P L_{j} \mid \mathcal{D}_{a}^{n, 1}\right) \\
& =\lim _{j \rightarrow \infty}\left\langle h\left(M_{A_{j}}\right) F\left(M_{A_{j}}\right) e, F\left(M_{A_{j}}\right) e\right\rangle \\
& \geq 0 \text {. }
\end{aligned}
$$

Thus $h(T) \geq 0$ which completes the proof of Reduction 2 .

Our final reduction rests on the observation that if $T$ is a finitely cyclic 2 -isometry possessing a cyclic $n$-tuple $\mu$ such that $(T, \mu)^{-}$is regular, then by Theorem 4.18 there exists a cyclic $n$-tuple $\gamma$ for $T$ such that $L=(T, \gamma)^{\wedge}$ is in Wiener-Hopf form with bounded intercept (in fact, smooth). Thus, we obtain the following.

Reduction 3. If (7.13) holds for all $T=M_{A}$ of covariance $\sigma$ where $A$ is an $n \times n$ matricial analytic DTO in Wiener-Hopf form with bounded intercept, then (7.13) holds for all $n$-cyclic regular 2-isometries of covariance $\sigma$.

We now are able to conclude the proof of Theorem 5.80 by observing that in light of the three reductions that it suffices to prove that (7.13) holds when $T=M_{A}, \operatorname{cov}(T)=\sigma$ 
and $A$ is an analytic DTO in Wiener-Hopf form with bounded intercept. By Theorem 6.20 , there exists a Hilbert space $\mathcal{K} \supseteq H_{A}^{2}$ and a Brownian unitary $B \in \mathcal{L}(\mathcal{K})$ of covariance $\sigma$ such that $H_{A}^{2}$ is invariant for $B$ and $T=B \mid H_{A}^{2}$. Thus, if $m \geq 1, h \in \mathbb{C}^{m, m}[x, y]$, and $h\left(c_{\sigma}\right) \geq 0$, then $h(B) \geq 0$ and so also by (7.2) $h(T) \geq 0$. This shows that (7.13) holds for $T$ and completes the proof of Theorem 5.80 .

\section{References}

[Ag1] Agler, J., Subjordan operators, Thesis, Indiana University, 1980.

[Ag2] Agler, J., "The Arveson extension theorem and coanalytic models," Integral Equations and Operator Theory 5 (1982), 608-631.

[Ag3] Agler, J., An Abstract Approach to Model Theory, Surveys of some recent results in operator theory Vol2, Pitman Research Notes in Mathematics Series; ISSN 0269-3674; 192, 1-24.

[Ag4] Agler, .J., "A Disconjugacy Theorem for "Toeplitz Operators", American Journal of Mathematics, 112(1990), 1-14.

[Ag5] Agler, .J., "Sub-Jordan Operators Bishop's Theorem, Spectral Inclusion and Spectral Sets", J. Operator Theory 7(1982), 373-395.

[Ag6] Agler, J., "Hypercontractions and Subnormality", J. Operator Theory 13(1985), 203217.

[Ag7] Agler, J., "Rational Dilation of an anmulus", Annals of Mathematics, 121(1985), $537-563$.

[AgSt] Agler, .J., Stankus, M. "m-Isometric Transformations Of Hilbert Space", Journal of Integral Equations and Operator Theory, 21(1995) \#4.

[Arv1] Arveson, W.B., "Subalgebras of $C^{*}$-algebras", Acta Math. 123(1969), 141-224.

[Arv2] Arveson, W.B., "Subalgebras of $C^{*}$-algebras II", Acta Math. 128(1972), 271-308.

[B] Beurling, A., "On two problems concerning Linear transformations in Hilbert space," Acta. Math. 81 (1949), 239-255.

[Ber-S] Berger, C.A., Shaw, B.L., "Self-commutators of multicyclic hyponormal operators are always trace class", B.A.M.S., 79(1973), 1193-1199.

[Bir-S] Birman, M. S., and Solomjak, M. Z., Spectral Theory of self-adjoint Operators in Hilbert Space, D. Reidel Publishing Company, Dordrecht/Boston/Lancaster/Tokyo, 1986.

[Br-R] de Branges, L., Rovnyak, J., Square Summable Power Series, New York, Holt, Rinehart and Winston, 1966. 
[C] Conway, John B., A Course in Functional Analysis, New York/Berlin/Heidelberg/ Tokyo, Springer-Verlag, 1985.

[Ca] Calkin, J.W., "Two-sided ideals and congruences in the ring of bounded operators in Hilbert space", Ann. of Math. (2) 42(1941), 839-873.

[C-F] Colojoară, I., Foias C., Theory of Generalized spectral Operators, New York, Gordon and Breach, 1968.

[C-P] Curto, R.E. and Putinar, M., "Existence of non-subnormal polynomially hyponormal operators", B.A.M.S., 25(1991) \#2, 373-378.

[F] Fillmore, P. A., Notes on operator theory, New York, Van Nostrand, 1970.

[Fr] Franks, E., Polynomially subnormal operator tuples, Thesis, University of California, San Diego, 1991.

[Ha] Halmos, P.R., A Hilbert space problem book, Princeton, D. Van Nostrand, 1970.

[H1] Helton, J. W., "Operators with a representation as multiplication by $x$ on a Sobolev space," Colloquia Math. Soc. Janos Bolyai 5, Hilbert Space Operators, Tihany, Hungary (1970), 279-287.

[H2] Helton, J. W., "Infinite dimensional Jordan operators and Sturm-Liouville conjugate point theory," Trans. Amer. Math. Soc. 170 (1972), 305-331.

[H3] Helton, J. W., Operator Theory, analytic functions, matrices, and electrical engineering, Conference Board of the Mathematical Sciences by the American Mathematical Society, 1987.

[Hel] Helson, H., Lectures on Invariant Subspaces, New York, Academic Press, 1964.

[M1] McCullough, S.A., 3-isometries, Thesis, University of California, San Diego, 1987.

[M2] McCullough, S.A., "SubBrownian Operators", J. Operator Theory, 22(1989) 2, 291305.

[M-G] Boutet de Monvel and V. Guillemin, The spectral theory of Toeplitz operators, Princeton University Press, Princeton N.J., University of Tokyo Press, Tokyo, 1981.

[N] Nielsen, O.A., "Direct Integral Theory," Lecture notes in pure and applied mathematics, vol. 61, Marcel Dekker, Inc, New York and Basel, 1980.

[P] Putnam, C.R., "An inequality for the area of hyponormal spectra", Math.Z., 116(1970), 323-330.

[R1] Richter, S., "Invariant subspaces of the Dirichlet shift," J. reine agnew. Math. 386 (1988), 205-220.

[R2] Richter, S., "A representation theorem for cyclic analytic two-isometries," Trans. Amer, Math. Soc., to appear. 
[Sa] Sarason, D., "Doubly shift-invariant spaces in $H^{2}$," J. Operator Theory 16 (1986), 75-97.

[Steg] Stegenga, D.A., "Multipliers of Dirichlet space", Ill. Jour. of Math. 24(1980), 113139.

[St] Stankus, M., Isosymmetric Linear Transformations On Complex Hilbert Space, Thesis, University of California, San Diego, 1993.

[Sti] Stinespring, W.F., "Positive functions on $C^{*}$-algebras", P.A.M.S. 6(1955), 211-216.

[Sz.-N,F] Sz.-Nagy. Foias, C., Harmonic analysis of operators in Hilbert space, North Holland. Amsterdam, 1970. 